\title{
DIFFERENTIAL OPERATORS ON AN AFFINE CURVE
}

\author{
S. P. SMITH and J. T. STAFFORD
}

[Received 11 November 1986]

\begin{abstract}
Let $X$ denote an irreducible affine algebraic curve over an algebraically closed field $k$ of characteristic zero. Denote by $\mathscr{D}_{X}$ the sheaf of differential operators on $X$, and $\mathscr{D}(X)=\Gamma\left(X, \mathscr{D}_{X}\right)$, the ring of global differential operators on $X$. The following is established:
\end{abstract}

THEOREM. $\mathscr{D}(X)$ is a finitely generated $k$-algebra, and a noetherian ring. Furthermore, $\mathscr{D}(X)$ has a unique minimal non-zero ideal $J$, and $\mathscr{D}(X) / J$ is a finite-dimensional $k$-algebra.

Let $\bar{X}$ denoted the normalisation of $X$, and $\pi: \tilde{X} \rightarrow X$ the projection map. The main technique is to compare $\mathscr{D}(X)$ with $\mathscr{D}(\bar{X})$.

THEOREM. The following are equivalent: (i) $\pi$ is injective, (ii) $\mathscr{D}(X)$ is a simple ring, (iii) $\mathscr{D}(X)$ is Morita equivalent to $\mathscr{D}(\bar{X})$, (iv) the categories $\mathscr{D}_{X}$-Mod and $\mathscr{D}_{\tilde{X}} \operatorname{Mod}$ are equivalent, $(\mathrm{v}) \mathrm{gr} \mathscr{D}(X)$ is noetherian, (vi) the global homological dimension of $\mathscr{D}(X)$ is 1 .

For higher-dimensional varieties the techniques produce examples of varieties $X$ for which $\mathscr{D}(X)$ is right but not left noetherian.

\section{Introduction}

0.1 . Throughout the paper $k$ will denote an algebraically closed field of characteristic zero, and all rings will be $k$-algebras. Further, unless we explicitly say otherwise, 'a variety $X$ ' will always mean 'an irreducible affine algebraic variety over $k$ ', and 'a curve' will be an 'irreducible affine algebraic curve over $k$ '. The ring of regular functions on a variety $X$ is denoted $O(X)$. Given a variety $X$, we will always write $\tilde{X}$ for the normalisation of $X$ and $\pi: \bar{X} \rightarrow X$ for the natural projection.

Given a variety $X$, we study $\mathscr{D}(X)$, the ring of global differential operators on $X$, as defined by Grothendieck $[9, \S 16.8 .1]$. If $X$ is non-singular, the structure of $\mathscr{D}(X)$ is particularly pleasant. Indeed, it follows from $[9, \S 16.11 .2]$ that $\mathscr{D}(X)$ is a finitely generated, noetherian $k$-algebra, and by [4, Chapter 3 , Theorem 2.5] is even a simple ring of finite global homological dimension. Most of these properties follow from the fact that the associated graded algebra, $\operatorname{gr} \mathscr{D}(X)$, formed by filtering $\mathscr{D}(X)$ by the order of the differential operators, is a regular, finitely generated commutative domain $[9, \S 16.11 .2]$.

The question therefore arises as to the structure of $\mathscr{D}(X)$ when $X$ is singular; for example, Malgrange [13] asks whether $\mathscr{D}(X)$ need be finitely generated and noetherian. The results for singular $X$ are scattered and partial, even when $X$ is a curve, and take a commutative point of view; see, for example, $[2,5,26]$. In this paper we study the structure of $\mathscr{D}(X)$, for $X$ a curve, from a non-commutative point of view and obtain strong results about the structure of $\mathscr{D}(X)$.

A.M.S. (1980) subject classification: 13N05, 14H20, 16A19, 16A33.

Proc. London Math. Soc. (3) 56 (1988) 229-259. 
0.2. The main results for curves in this paper can be encapsulated in the following two theorems. Let $X$ be a curve.

THEOREM A. $\mathscr{D}(X)$ is a finitely generated (right and left) noetherian $k$-algebra, for which the endomorphism ring of every simple $\mathscr{D}(X)$-module is equal to $k$. Further, $\mathscr{D}(X)$ has a unique minimal non-zero ideal $J$, and $\mathscr{D}(X) / J$ is a finite-dimensional $k$-algebra.

THEOREM B. The following are equivalent:

(a) $\pi$ is injective;

(b) $\mathscr{D}(X)$ is a simple ring (and Morita equivalent to $\mathscr{D}(\tilde{X})$ );

(c) $\operatorname{gl} \cdot \operatorname{dim} \mathscr{D}(X)=1$;

(d) $\operatorname{gr} \mathscr{D}(X)$ is a finitely generated k-algebra (equivalently, $\operatorname{gr} \mathscr{D}(X)$ is noetherian).

Theorem A has been independently obtained (with a proof very similar to ours) by J. Muhasky [16].

0.3. The key idea in the proof of these results is to identify canonically $\mathscr{D}(X)$, and $\mathscr{D}(\tilde{X})$, with subalgebras of $\mathscr{D}(K)$ where $K$ is the fraction field of $\mathscr{O}(X)$. It then follows that $\mathscr{D}(\tilde{X}, X)$, the module of differential operators from $O(\tilde{X})$ to $\mathscr{O}(X)$, embeds in both $\mathscr{D}(X)$ and $\mathscr{D}(\tilde{X})$. Furthermore,

$$
\mathscr{D}(X) \subseteq T:=\operatorname{End}_{\mathscr{D}(\tilde{X})} \mathscr{D}(\tilde{X}, X) \subseteq \mathscr{D}(K) .
$$

The interplay between these five objects forms the main theme of this paper. Thus, for example, Theorem A follows easily from the fact that $\mathscr{D}(\tilde{X}, X)$ is a left ideal of both $T$ and $\mathscr{D}(X)$. This result is proved in $\S 2$, but see also (4.2). Some special cases of Theorem A appear in [5] and [26].

The crucial result in the proof of Theorem B is that $\pi$ is injective if and only if $\mathscr{D}(X)=T$. However, the proofs of the various equivalences are more involved than the proof of Theorem $A$, and appear in $\S \S 3$ and 4 . When $\pi$ is not injective we cannot give a complete description of the structure of $\mathscr{D}(X)$. However, Theorem 4.11 does give a characterisation of $\mathscr{D}(X)$ as an endomorphism ring, $\mathscr{D}(X) \cong \operatorname{End}_{\mathscr{D}(Y)} \mathscr{D}(Y, X)$, where $Y$ is a (singular) curve for which $\mathscr{D}(Y)$ can be explicitly described.

0.4. Despite the fact that, by Theorem $\mathrm{A}, \mathscr{D}(X)$ has only finitely many prime ideals, and even has the descending chain condition on two-sided ideals, it is still possible for $\mathscr{D}(X)$ to have infinitely many ideals, as is shown in $\S 5$.

0.5 . In $\S 6$ we briefly consider the case of differential operators on a (singular) projective curve $C$, and show that if $\pi: \tilde{C} \rightarrow C$ is injective then $\mathscr{D}_{C}$-Mod and $\mathscr{D}_{\bar{C}}$-Mod are equivalent (here $\mathscr{D}_{C}$-Mod is the category of sheaves of quasicoherent $\mathscr{D}_{C}$-modules).

0.6. Finally, we consider the structure of $\mathscr{D}(X)$ where $X$ is a variety of dimension at least 2. Here, the question of Malgrange mentioned earlier has a negative answer-the first example being the normal surface given by $X_{1}^{3}+X_{2}^{3}+$ 
$X_{3}^{3}=0$ [3]. For this variety, $\mathscr{D}(X)$ is neither noetherian nor finitely generatedthe proof depends on detailed computations concerning vector bundles on an elliptic curve. In $\$ 7$ we consider the case when $X$ is a singular variety whose normalisation $\tilde{X}$ is non-singular; in this case the techniques for curves may still be applied. In this situation $\mathscr{D}(X)$ is frequently not noetherian; indeed, we have

THEOREM C. If $X$ is non-singular in codimension 1 , then $\mathscr{D}(X)$ is not left noetherian. In contrast, if $X$ has only finitely many singularities, then $\mathscr{D}(X)$ is right noetherian and a finitely generated $k$-algebra.

0.7. The starting point for this investigation was an example of I. Musson, who gave an explicit description of $\mathscr{D}(X)$ for the cusp $x^{2}=y^{3}$. He showed that $\mathscr{D}(X)$ coincided with the explicit description (given in [22]) of

$$
\operatorname{End}_{A_{1}}\left(x^{2} A_{1}+(x \partial-1) A_{1}\right)
$$

where $A_{1}=k[x, \partial]=\mathscr{D}\left(\mathbf{A}^{1}\right)$ is the first Weyl algebra and $\partial=\partial / \partial x$. This curious coincidence motivated the authors' interest in this subject, and we would like to thank Musson for sharing his example with us. His work appears in [17].

0.8. This research was conducted while the first author was supported by a (British) SERC Research Assistantship. Some of the research was done while the second author was visiting the University of Washington, Seattle and supported in part by an NSF grant. The authors would like to express their gratitude to all three organisations.

\section{Generalities on differential operators}

1.1. In this section we give definitions and results on differential operators which are required in the main part of the paper. While most of the results are well known there is not an appropriate reference for our needs.

1.2. Let $A$ be a commutative $k$-algebra, and let $M$ and $N$ be $A$-modules. Give $\operatorname{Hom}_{k}(M, N)$ the structure of an $A \otimes_{k} A$-module by defining $((a \otimes b) \theta)(m)=$ $a \theta(b m)$ for $a, b \in A, \theta \in \operatorname{Hom}_{k}(M, N)$, and $m \in M$. Define $\mu: A \otimes A \rightarrow A$ to be the multiplication map, $\mu(a \otimes b)=a b$. Write $J_{A}$ (or simply $J$ ) for ker $\mu$.

Definition. The space of $k$-linear differential operators from $M$ to $N$ of order at most $n$ is defined by

$$
\mathscr{D}_{A}^{n}(M, N)=\left\{\theta \in \operatorname{Hom}_{k}(M, N) \mid J^{n+1} \theta=0\right\},
$$

where $J^{0}=A \otimes_{k} A$. Set $\mathscr{D}_{A}(M, N):=\bigcup_{n=0}^{\infty} \mathscr{D}_{A}^{n}(M, N)$. The subscript $A$ in $\mathscr{D}_{A}($, ) will be dropped whenever convenient. Write $\mathscr{D}(M)=\mathscr{D}(M, M)$.

1.3. The reader is referred to $[9, \S 16],[11]$, and $[\mathbf{2 5}$, Chapters 8,13$]$ for the following results and observations. Although some of the results hold more generally, we assume that $A \otimes_{k} A$ is noetherian, throughout (1.3).

(a) The ideal $J$ is generated by $\{1 \otimes a-a \otimes 1 \mid a \in A\}$. We shall write $[\theta, a]:=(1 \otimes a-a \otimes 1) \theta=\theta a-a \theta \quad$ for $\quad a \in A, \quad \theta \in \operatorname{Hom}_{k}(M, N)$. Thus $\mathscr{D}^{0}(M, N)=\operatorname{Hom}_{A}(M, N)$, and $\mathscr{D}(M, N)=0$ if and only if $\operatorname{Hom}_{A}(M, N)=0$. 
Given the above description of $J$, one may alternatively define $\mathscr{D}^{n}(M, N)$ inductively by $\mathscr{D}^{-1}(M, N)=0$ and, for $n \geqslant 0$,

$$
\mathscr{D}^{n}(M, N)=\left\{\theta \in \operatorname{Hom}_{k}(M, N) \mid[\theta, a] \in \mathscr{D}^{n-1}(M, N) \text { for all } a \in A\right\} .
$$

(b) One verifies for all $n, m \in \mathbf{N}$, that the composition of maps gives $\mathscr{D}^{n}(M, N) \times \mathscr{D}^{m}(L, M) \rightarrow \mathscr{D}^{n+m}(L, N)$. Hence in the special case when $L=M=$ $N$, it follows that $\mathscr{D}(M)$ is a $k$-subalgebra of $\operatorname{End}_{k} M$. Likewise one sees that $\mathscr{D}(M, N)$ becomes a $\mathscr{D}(N)-\mathscr{D}(M)$ bimodule where the module action is given by the composition of maps. Finally, the above gives a $\mathscr{D}(N)-\mathscr{D}(L)$ bimodule map $\mathscr{D}(M, N) \otimes_{\mathscr{C D}(M)} \mathscr{D}(L, M) \rightarrow \mathscr{D}(L, N)$.

(c) The above also shows that $\mathscr{D}(M,-)$ and $\mathscr{D}(-, M)$ are functors from $A$-Mod to Mod- $\mathscr{D}(M)$ (the category of right $\mathscr{D}(M)$-modules) and to $\mathscr{D}(M)$-Mod (the category of left $\mathscr{D}(M)$-modules) respectively. It follows from the fact that $\operatorname{Hom}_{k}(M,-)$ and $\operatorname{Hom}_{k}(-, M)$ are left exact that $\mathscr{D}(M,-)$ and $\mathscr{D}(-, M)$ are also left exact. However, they need not be right exact. But on a split exact sequence, both $\mathscr{D}(M,-)$ and $\mathscr{D}(-, M)$ are exact.

(d) For each $n \geqslant 0$, write $P_{A}^{n}=A \otimes_{k} A / J_{A}^{n+1}$ (we drop the subscript when convenient). It is immediate from the definition in (1.2) that

$$
\mathscr{D}^{n}(M, N) \cong \operatorname{Hom}_{A \otimes A}\left(P^{n}, \operatorname{Hom}_{k}(M, N)\right)
$$

and then from the adjointness of $\otimes$ and Hom, one obtains

$$
\mathscr{D}^{n}(M, N) \cong \operatorname{Hom}_{A}\left(P^{n} \otimes_{A} M, N\right)
$$

where $P^{n} \otimes_{A} M$ is formed by giving $P^{n}$ the right $A$-module structure through $A \rightarrow 1 \otimes A \subseteq A \otimes A$, and $P^{n} \otimes_{A} M$ is considered as a left $A$-module through $A \rightarrow A \otimes 1 \subseteq A \otimes A$. It is then relatively straightforward to show that if $S \subset A$ is a multiplicatively closed subset and $M$ is either finitely presented, or an $A_{S}$-module, then $A_{S} \otimes_{A} \mathscr{D}_{A}(M, N) \cong \mathscr{D}_{A_{S}}\left(M_{S}, N_{S}\right)$. Finally, the above description of $\mathscr{D}^{n}(M, N)$ gives

$$
\mathscr{D}(M, N) \cong \lim _{\rightarrow} \operatorname{Hom}_{A}\left(P^{n} \otimes_{A} M, N\right),
$$

where the direct limit is the obvious one coming from the natural maps $P^{n+1} \rightarrow P^{n}$.

(e) Suppose that each $P^{n}$ is a projective $A$-module (on the left). Then, by (d),

$$
\mathscr{D}(A, N)=\lim _{\longrightarrow} \operatorname{Hom}_{A}\left(P^{n}, N\right)=\underline{\lim _{\longrightarrow}} N \otimes_{A}\left(P^{n}\right)^{*}
$$

where $\left(P^{n}\right)^{*}=\operatorname{Hom}_{A}\left(P^{n}, A\right)$. Because direct limits commute with tensor products, this gives $\mathscr{D}(A, N) \cong N \bigotimes_{A}\left(\lim \left(P^{n}\right)^{*}\right)$. But putting $N=A$, gives

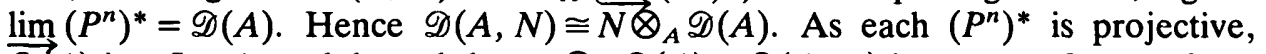
$\vec{D}(A)$ is a flat $A$-module and thus $-\bigotimes_{A} \mathscr{D}(A)=\mathscr{D}(A,-)$ is an exact functor from $A$-Mod to Mod- $\mathscr{D}(A)$. It is also a faithful functor, since if $N \neq 0$ then $\operatorname{Hom}_{A}(A, N) \neq 0$, and thus $\mathscr{D}(A, N) \neq 0$. Note that if $I$ is an ideal of $A$ then $\mathscr{D}(A, I) \cong I \bigotimes_{A} \mathscr{D}(A) \cong I \mathscr{D}(A)$ where the last isomorphism is obtained by viewing $A$ as a subalgebra of $\mathscr{D}(A)$. Similarly, $\mathscr{D}(A, A / I) \cong \mathscr{D}(A) / I \mathscr{D}(A)$.

(f) If $X$ is a non-singular variety over $k$, and $A$ is any localisation of $O(X)$, the ring of regular functions, then each $P_{A}^{n}$ is projective and so (e) applies.

(g) The $k$-subspaces $\mathscr{D}^{n}(A)$ of $\mathscr{D}(A)$ filter $\mathscr{D}(A)$, and it is easy to check that gr $\mathscr{D}(A)$, the associated graded algebra, is commutative. 
1.4. We now concentrate on the case when $A=\mathscr{O}(X)$, the co-ordinate ring of a non-singular variety $X$. We shall write $\mathscr{D}(X)$ for $\mathscr{D}(\mathscr{O}(X))$. Many of the following facts may be found in [4].

(a) The ring $\mathscr{D}(X)$ is a finitely generated $k$-algebra, generated by $\mathscr{O}(X)$ and $\operatorname{Der}_{k} \mathcal{O}(X)$, the module of $k$-linear derivations on $\mathscr{O}(X)$. Furthermore, $\operatorname{gr} \mathscr{D}(X) \cong$ $\mathcal{O}\left(T^{*} X\right)$ where $T^{*} X$ denotes the cotangent bundle (which is also a non-singular variety). In particular, $\operatorname{gr} \mathscr{D}(X)$ is a noetherian domain, and hence $\mathscr{D}(X)$ is also a (right and left) noetherian domain.

(b) We shall denote by $\mathscr{O}_{X}$ the sheaf of regular functions on $X$, and by $\mathscr{D}_{X}$ the sheaf of differential operators on $X$. The stalks at a point $x \in X$ will be denoted by $\mathscr{O}_{X, x}$ and $\mathscr{D}_{X, x}$ respectively. The stalk $\mathscr{D}_{X, x}$ has a structure similar to that of the Weyl algebra, $\mathscr{D}\left(\mathbf{A}^{n}\right)$, since $\operatorname{Der}_{k} \mathscr{O}_{X, x}$ is the free $\mathscr{O}_{X, x}$-module generated by derivations $\partial_{1}, \ldots, \partial_{n}$ which satisfy $\partial_{i}\left(t_{j}\right)=\delta_{i j}$ where the $t_{j}$ are chosen to be a system of parameters for the $n$-dimensional regular local ring $\mathcal{O}_{X, x}$. Hence $\mathscr{D}_{X, x} \cong \mathcal{O}_{X, x}\left[\partial_{1}, \ldots, \partial_{n}\right]$.

(c) By imitating the proof for the Weyl algebra, one shows that $\mathscr{D}_{X, X}$ is a simple ring, and consequently $\mathscr{D}(X)$ is a simple ring.

(d) The endomorphism ring of a simple $\mathscr{D}(X)$-module is precisely $k$, and for an artinian $\mathscr{D}(X)$-module $M$, End $\mathrm{E}_{\mathscr{D}(X)} M$ is a finite-dimensional $k$-vector space (this follows from Quillen's Lemma [18]).

(e) The global homological dimension of $\mathscr{D}(X)$ is finite; indeed gl.dim $\mathscr{D}(X)=$ $\operatorname{dim} X$. The Krull dimension (in the sense of Rentschler-Gabriel) of $\mathscr{D}(X)$ also equals $\operatorname{dim} X$. The Gelfand-Kirillov dimension of $\mathscr{D}(X)$ equals $2 \operatorname{dim} X$ [20].

(f) If $M$ is a finitely generated $\mathscr{D}(X)$-module then $d(M)$ denotes the Krull dimension of the associated graded module $\operatorname{gr} M$. If $M \neq 0$, then $d(M) \geqslant \operatorname{dim} X$, and if equality occurs then $M$ is said to be holonomic. If $M$ is a holonomic $\mathscr{D}(X)$-module, then $M$ is of finite length.

(g) If $\mathfrak{m}$ is a maximal ideal of $\mathscr{O}(X)$, then $\mathfrak{m} \mathscr{D}(X)$ is a maximal right ideal of $\mathscr{D}(X)$. This is because $\operatorname{gr}(\mathfrak{m} \mathscr{D}(X))=\mathfrak{m} \operatorname{gr} \mathscr{D}(X)$ is a prime ideal of $\operatorname{gr} \mathscr{D}(X)$ such that the Krull dimension of $\operatorname{gr} \mathscr{D}(X) / \mathfrak{m} \operatorname{gr} \mathscr{D}(X)$ equals $\operatorname{dim} X$. So any proper factor module of $\mathscr{D}(X) / \mathfrak{m} \mathscr{D}(X)$ has associated graded module of Krull dimension strictly less than $\operatorname{dim} X$. But by (f) the only such module is zero.

\subsection{Definition. Let $J$ be a right ideal of a ring $R$. The idealiser of $J$ in $R$ is}

$$
\mathbf{I}_{R}(J):=\{x \in R \mid x J \subseteq J\} .
$$

The natural importance of the idealiser arises from the fact that it is the largest subring of $R$ in which $J$ is a two-sided ideal, and furthermore $\mathbf{I}(J) / J \cong \operatorname{End}_{R}(R / J)$.

One useful observation in this context is the following. Let $X$ be a non-singular variety, and $I$ a right ideal of $\mathscr{D}(X)$ such that $\mathscr{D}(X) / I$ is of finite length. Then $\mathbf{I}(I) / I$ is finite-dimensional by (1.4d)). This is an essential ingredient in the proof of Theorem A (see also (7.3)).

1.6. The following result is often stated in the literature but never (so it seems) proved. We give a proof partly to fill this gap, and partly to illustrate how the elementary considerations in (1.3) may be used.

Notation. If $D \in \mathscr{D}(A)$ and $a \in A$, there is a possibility of confusion between $D(a)$, through $D$ acting on $A$, and $D a$ the product in $\mathscr{D}(A)$. We shall therefore 
write $D * a$ for $D(a)$ in order to avoid confusion. Thus, if $I$ is an ideal of $A, D * I$ denotes $D(I)$, the image of $I$ under the action of $D$ on $A$.

Proposition. Let $X$ be a non-singular variety, and $Y$ a closed subvariety defined by the ideal $I$ of $\mathcal{O}(X)$. Then

$$
\mathscr{D}(Y) \cong \mathbf{I}(I \mathscr{D}(X)) / I \mathscr{D}(X) .
$$

Proof. Write $A=\mathscr{O}(X)$. There is a natural map $\varphi: \mathbf{I}(I \mathscr{D}(A)) \rightarrow \mathscr{D}(Y)$ given as follows. If $D \in \mathbf{I}(I \mathscr{D}(A))$ then $D I \subseteq I \mathscr{D}(A)$, and hence

$$
D * I=D I * A \subseteq I \mathscr{D}(A) * A=I .
$$

Thus $D$ induces $D^{\prime}: A / I \rightarrow A / I$. Since $\mathscr{D}(Y)=\mathscr{D}(A / I, A / I)$, we define $\varphi(D)=$ $D^{\prime}$. The kernel is

$$
\text { ker } \varphi=\{D \in \mathscr{D}(A) \mid D * A \subseteq I\}=\mathscr{D}(A, I)=I \mathscr{D}(A),
$$

where the final equality is given by $(1.3(\mathrm{e})$, (f)). Hence $\varphi$ induces an injective map $\mathbf{I}(I \mathscr{D}(A)) / I \mathscr{D}(A) \rightarrow \mathscr{D}(Y)$ given by $D \rightarrow D^{\prime}$.

We construct an inverse to show it is surjective. Apply the left exact functor $\mathscr{D}(-, A / I)$ to the exact sequence $0 \rightarrow I \rightarrow A \rightarrow A / I \rightarrow 0$. One obtains

$$
0 \rightarrow \mathscr{D}(Y) \rightarrow \mathscr{D}(A, A / I) \rightarrow \mathscr{D}(I, A / I) .
$$

However, by $(1.3(\mathrm{e}),(\mathrm{f})), \mathscr{D}(A, A / I)=\mathscr{D}(A) / I \mathscr{D}(A)$ and hence the injection $\mathscr{D}(Y) \rightarrow \mathscr{D}(A, A / I)$ is the required inverse. This completes the proof.

\section{The noetherian property for differential operators on a curve}

2.1. The aim of this section is to show that many of the properties of $\mathscr{D}(Z)$, for $Z$ non-singular, as for example illustrated by (1.4), also hold for $\mathscr{D}(X)$ when $X$ is a curve. Thus, for example, we show that $\mathscr{D}(X)$ is a finitely generated, noetherian $k$-algebra. The proof of this is remarkably easy, the key idea being to take the normalisation $\bar{X}$ of $X$, and canonically identify $\mathscr{D}(X)$ with a subring of the division ring of fractions of $\mathscr{D}(\bar{X})$. Since this method is more widely applicable, we begin in a more general situation.

2.2. Let $X$ be a variety, and $Y$ a variety such that there exist morphisms $\tilde{X}^{\psi} \rightarrow Y^{\varphi} \rightarrow X$ such that $\psi$ is surjective and $\varphi \psi=\pi$, where $\pi: \tilde{X} \rightarrow X$ is the normalisation morphism. (In this section our applications will be to the case $Y=\tilde{X}$, but a more general $Y$ will be required in $\S 4$.) In terms of regular functions $\mathcal{O}(X) \subseteq \mathcal{O}(Y) \subseteq \mathcal{O}(\tilde{X})$, and $\mathcal{O}(\tilde{X})$ is the integral closure of $\mathcal{O}(X)$ in its field of fractions $K=k(X)$.

Since $\mathscr{D}(K)=K \otimes_{O(Y)} \mathscr{D}(Y)$ by $(1.3(\mathrm{~d}))$, we may identify $\mathscr{D}(Y)$ with its image in $\mathscr{D}(K)$. In other words, in the notation of (1.6),

$$
\mathscr{D}(Y)=\{D \in \mathscr{D}(K) \mid D * f \in \mathscr{O}(Y) \text { for all } f \in \mathscr{O}(Y)\} .
$$

Of course $Y$ may be taken to be either $X$ or $\tilde{X}$ in this identification. Thus the three algebras $\mathscr{D}(X), \mathscr{D}(Y), \mathscr{D}(\tilde{X})$ can (and always will) be viewed as subalgebras of $\mathscr{D}(K)$. 
2.3. If $A \subset B$ are commutative $k$-algebras then we write

$$
\mathscr{D}(B, A)=\{D \in \mathscr{D}(B) \mid D * f \in A \text { for all } f \in B\} .
$$

Thus, $\mathscr{D}(B, A)$ is a right ideal of $\mathscr{D}(B)$. If the identifications of $(2.2)$ are made, with $B=\mathscr{O}(Y)$ and $A=\mathscr{O}(X)$, then $\mathscr{D}(B, A)$ is also a left ideal of $\mathscr{D}(A)$. In this situation we shall write $\mathscr{D}(Y, X):=\mathscr{D}(\mathscr{O}(Y), \mathscr{O}(X))$. We remark that $\mathscr{D}(B, A)$ as defined here is not necessarily equal to $\mathscr{D}_{A}(B, A)$ as defined in (1.2). As it does not affect the results of this section we delay discussion of this point until (2.7).

Since $\mathscr{D}(K)$ is a noetherian domain, Goldie's Theorem implies that it has a division ring of fractions, say $Q$, and this is therefore also the ring of fractions of $\mathscr{D}(X)$ and $\mathscr{D}(Y)$. Write $P=\mathscr{D}(Y, X)$ and set $T=\{q \in Q \mid q P \subseteq P\}$. Thus $T \cong \operatorname{End}_{\mathscr{D}(Y)} P$. As $P$ is a left ideal of $\mathscr{D}(X)$, it follows that $\mathscr{D}(X) \subseteq T$. Furthermore, $T \subseteq \mathscr{D}(K)$. To see this, observe that the conductor $I=$ $\mathrm{Ann}_{\mathscr{O}(X)}(\mathscr{O}(Y) / \mathscr{O}(X))$ is non-zero. Obviously, $I \mathscr{D}(Y) \subseteq P$, and so if $q \in T$ then $q I \subseteq P \subseteq \mathscr{D}(Y)$. In particular, for any $0 \neq a \in I$ we have $q \in \mathscr{D}(Y) a^{-1} \subseteq \mathscr{D}(K)$, as required.

A diagram illustrates the relationships determined in the last two paragraphs:

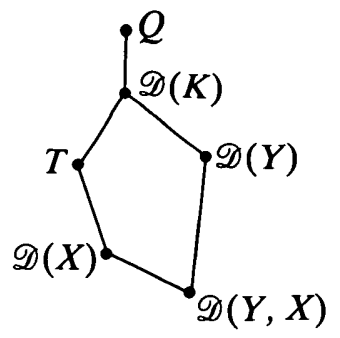

2.4. Before we give the main result of this section, one further definition is required.

Two rings $R$ and $S$ are Morita equivalent if there exists a finitely generated projective (right) $R$-module $P$ that is a generator in Mod- $R$, and such that $S \cong \operatorname{End}_{R} P$. While many properties of rings (such as being simple or noetherian) are known to be Morita invariant, the following does not appear to be so well known although it is essentially in [15]. (Standard facts about Morita equivalence can be found, for example, in [1, Chapter 6].)

Lemma. Suppose that $S$ and $R$ are Morita equivalent $k$-algebras. If $R$ is a finitely generated $k$-algebra, then so is $S$.

Proof. There exist $n \in \mathbf{N}$, and an idempotent $e \in M=M_{n}(R)$, the $n \times n$ matrix ring, such that $e M e \cong S$ and $M=M e M$ (see [1, Corollary 22.7]). Certainly $M$ is finitely generated, and it therefore follows from [15, Corollary 1] that $S$ is also finitely generated.

2.5. Theorem. Let $X$ be a curve. Then $\mathscr{D}(X)$

(a) is (right and left) noetherian;

(b) is a finitely generated $k$-algebra; 
(c) has (Gabriel-Rentschler) Krull dimension 1;

(d) has Gelfand-Kirillov dimension 2;

(e) has the property that $\operatorname{End}_{\mathscr{D}(X)} M=k$, for every simple (right or left) $\mathscr{D}(X)$-module $M$.

Proof. Make the identifications of (2.2) and (2.3). By (1.4) all the above properties hold for $\mathscr{D}(\tilde{X})$. Note that $P=\mathscr{D}(\tilde{X}, X)$ is a projective $\mathscr{D}(\tilde{X})$-module by $(1.4(\mathrm{e}))$, and a generator in $\operatorname{Mod}-\mathscr{D}(\tilde{X})$ by $(1.4(\mathrm{c}))$. Thus $T=\operatorname{End}_{\mathscr{D}(\bar{X})} P$ is Morita equivalent to $\mathscr{D}(\tilde{X})$ and the above properties hold for $T$ (see [1, pp. 252, 258] and Lemma 2.4).

We now appeal to [20]. Note that $\mathscr{D}(X)$ contains the left ideal $P$ of $T$. In the language of [20] this means that $\mathscr{D}(X)$ and $T$ are equivalent orders. Thus all but part (d) of the theorem follow from the previous paragraph and [20, Theorem 3 (iii) and Corollary 4]. Finally, $P$ is a $\mathscr{D}(\tilde{X})-\mathscr{D}(X)$ bimodule which is finitely generated on both sides. Thus (d) follows from [12, Corollary 3] combined with the statement in $(1.4(\mathrm{e}))$ that $\mathrm{GK}-\operatorname{dim} \mathscr{D}(\tilde{X})=2$.

2.6. In [5, Corollary 3.6] and [26, II, Corollaire 4] the following is proved. Let $Z$ be an analytic curve irreducible at a point $z \in Z$. Denote by $\mathscr{O}_{z, z}$ the ring of germs of analytic functions at $z$, and by $\mathscr{D}_{z, z}$ the ring of differential operators on $\mathscr{O}_{z, z}$. Then $\mathscr{D}_{z, z}$ is noetherian and generated by $\mathscr{O}_{z, z}$ and a finite number of differential operators. The proofs in [5] and [26] are sufficiently computational to allow one to compute generators for $\mathscr{D}_{z, z}$. They are also able to show that gr $\mathscr{D}_{z, z}$ is a finitely generated $O_{z, z}$-algebra. The proof of (2.5) may also be applied to the analytic case to give the results on $\mathscr{D}_{Z, z}$ just mentioned. We will return to the question of whether $\mathrm{gr} \mathscr{D}(X)$ is finitely generated in (3.9).

2.7. Consider the problem mentioned in (2.3), namely that for $k$-algebras $A \subset B$, the objects $\mathscr{D}(B, A)$ as defined in (2.3) and $\mathscr{D}_{A}(B, A)$ defined in (1.2) need not be the same. For example, take $A=k\left[t^{2}\right]$ and $B=k[t]$. It is easy to check that $\mathscr{D}(B, A)=0$, whereas $\mathscr{D}_{A}(B, A) \neq 0$. However, the only occasions on which we use $\mathscr{D}(B, A)$ are covered by the following lemma and so the two objects coincide. Thus the notational confusion should cause no problems.

Lemma. Let $A, B$ be domains such that $A \subset B \subset$ Fract $A$. Then $\mathscr{D}(B, A)=$ $\mathscr{D}_{A}(B, A)$. In particular, if $S \subset A$ is multiplicatively closed, then $\mathscr{D}\left(B_{S}, A_{S}\right) \simeq$ $A_{S} \otimes_{A} \mathscr{D}(B, A)$.

Proof. Note that under the embedding $A \bigotimes_{k} A \subset B \bigotimes_{k} B$, we have $J_{A} \subseteq J_{B}$ in the notation of (1.2). It follows that $J_{A}^{n} \subseteq J_{B}^{n}$, and so $\mathscr{D}(B, A) \subseteq \mathscr{D}_{A}(B, A)$. For the reverse inclusion, note first that if $K=$ Fract $A$, then $K \otimes_{A} B \cong K$, and since $A$ is a domain, $\mathscr{D}_{A}(B, A)$ is a torsion-free left $A$-module. Thus (1.3(d)) implies that

$$
\mathscr{D}_{A}(B, A) \subseteq K \otimes_{A} \mathscr{D}_{A}(B, A)=\mathscr{D}_{K}(K, K)=\mathscr{D}(K) .
$$

Thus, if $D \in \mathscr{D}_{A}(B, A)$ then $J_{K}^{n} D=0$ for some $n$, whence $J_{B}^{n} D=0$. Thus $D \in \mathscr{D}(B)$, and so $\mathscr{D}_{A}(B, A) \subseteq \mathscr{D}(B, A)$. The final sentence of the lemma follows by applying (1.3(d)) again. 
2.8. We understand that $\mathrm{J}$. Muhasky [16] has also (and independently) obtained Theorem 2.5, with similar techniques.

\section{When is $\mathscr{D}(X)$ simple, for $X$ a curve?}

3.1. Theorem 2.5 shows, for a curve $X$, that many of the properties of $\mathscr{D}(\bar{X})$ also hold for $\mathscr{D}(X)$. Since $\mathscr{D}(\tilde{X})$ is a simple ring by $(1.4(\mathrm{c}))$ and $\mathscr{D}(X)$ is contained in the simple ring $T=\operatorname{End}_{\mathscr{D}(\tilde{X})} \mathscr{D}(\tilde{X}, X)$, it is natural to ask whether $\mathscr{D}(X)$ is simple. We answer this question completely in this section by showing that $\mathscr{D}(X)$ is simple if and only if $\pi: \bar{X} \rightarrow X$ is injective. In fact, rather more is true, in that if $\pi$ is injective then $\mathscr{D}(X)=T$, and hence is Morita equivalent to $\mathscr{D}(\tilde{X})$. As an application we show that this same condition also determines when $\operatorname{gr} \mathscr{D}(X)$ is a finitely generated $k$-algebra (Theorem 3.12 ).

3.2. The first two results show that the simplicity of $\mathscr{D}(X)$ can be rephrased in terms of the action of $\mathscr{D}(\tilde{X}, X)$ on $\mathscr{O}(\bar{X})$. These elementary observations will prove useful later, so we assume that the situation is as in (2.2).

Lemma (Notation (2.2) and (2.3)). Suppose that $\mathscr{D}(Y, X) * \mathscr{O}(Y)=\mathscr{O}(X)$. Then $\mathscr{D}(X)=T=\operatorname{End}_{\mathscr{D}(Y)} \mathscr{D}(Y, X)$.

Proof. By (2.3), $\mathscr{D}(X) \subseteq T \subseteq \mathscr{D}(K)$. Let $D \in T$, and $f \in \mathscr{O}(X)$. Then $f \in$ $\mathscr{D}(Y, X) * \mathscr{O}(Y)$. Thus

$$
D * f \in D \mathscr{D}(Y, X) * \mathscr{O}(Y) \subseteq \mathscr{D}(Y, X) * \mathscr{O}(Y) \subseteq \mathscr{O}(X)
$$

(notice that $D * f$ is well defined because $D \in \mathscr{D}(K)$ ). Consequently $D \in \mathscr{D}(X)$, as required.

3.3. Proposition. Write $P=\mathscr{D}(\tilde{X}, X)$. Consider the following statements:

(a) $\mathscr{D}(X)$ is a simple ring;

(b) $\mathscr{D}(\tilde{X}, X) * \mathscr{O}(\tilde{X})=\mathscr{O}(X)$;

(c) $\mathscr{D}(X)=\operatorname{End}_{\mathscr{D}(\bar{X})} P$.

Then (a) $\Rightarrow$ (b) $\Rightarrow$ (c). Furthermore, if $X$ is a curve, then all three statements are equivalent to

(d) $\mathscr{D}(X)$ and $\mathscr{D}(\tilde{X})$ are Morita equivalent.

Proof. If $P * \mathscr{O}(\tilde{X}) \neq \mathscr{O}(X)$ then $\mathscr{O}(X) /(P * \mathscr{O}(\tilde{X}))$ is a non-zero left $\mathscr{D}(X)$ module. Its annihilator in $\mathscr{D}(X)$ contains $P$, and so $\mathscr{D}(X)$ is not a simple ring. Hence (a) implies (b). That (b) implies (c) is just Lemma 3.2.

For the rest of the proof let $X$ be a curve. As remarked in the proof of Theorem 2.5, $\mathscr{D}(\tilde{X})$ is Morita equivalent to $T=\operatorname{End}_{\mathscr{D}_{(\tilde{X})}} P$. Hence (c) implies (a), because simplicity is a Morita invariant by [1, Corollary 21.12]. Finally, if (c) holds then $\mathscr{D}(X)$ and $\mathscr{D}(\tilde{X})$ are Morita equivalent, so (c) implies (d). Conversely, if (d) holds then $\mathscr{D}(X)$ is simple because $\mathscr{D}(\tilde{X})$ is simple. Thus (d) implies (a).

3.4. We now apply (3.3) to the case when $X$ is a curve. 
THEOREM. Let $X$ be a curve, and suppose that $\pi: \tilde{X} \rightarrow X$ is injective. Then $\mathscr{D}(X)$ is a simple ring, Morita equivalent to $\mathscr{D}(\tilde{X})$.

REMARK. The hypothesis that $\pi$ is injective is equivalent to saying that each maximal ideal of $\mathcal{O}^{\prime}(X)$ is contained in a unique maximal ideal of $\mathcal{O}(\tilde{X})$.

Proof. By (3.3), it suffices to prove that $\mathscr{D}(\tilde{X}, X) * \mathscr{O}(\tilde{X})=\mathscr{O}(X)$. The proof will take place locally, as this allows us to write down some specific differential operators. To avoid some notation, write $A=\mathscr{O}(X)$ and $B=\mathscr{O}(\bar{X})$.

Let $M$ be a maximal ideal of $A$, and $Q$ the unique maximal ideal of $B$ containing $M$; thus $M=Q \cap A$. Write $S=A \backslash M$. Then $B_{S}=B_{Q}$ where $B_{S}$ (respectively $B_{Q}$ ) is obtained by inverting $S$ (respectively $B \backslash Q$ ). Set $\mathfrak{m}=Q B_{Q}$. As $B$ is integrally closed of dimension $1,\left(B_{Q}, \mathfrak{m}\right)$ is a regular local ring, and $\mathrm{m}$ is a principal ideal; say, $\mathrm{m}=t B_{Q}$ for some $t \in B_{Q}$. By [10, Chapter II, Theorem 8.8] the module of Kahler differentials $\Omega_{B_{Q}}$ is the free $B_{Q}$-module generated by $d t$, and $\operatorname{Der} B_{Q}$ is the free $B_{Q}$-module generated by a derivation $\partial$ such that $\partial * t=1$ (notation (1.6)). Think of $\partial$ as $\partial / \partial t$.

Write $I=\operatorname{Ann}_{A}(B / A)$. Thus $I B_{Q} \subseteq A_{S}$ and as the ideals of $B_{Q}$ are just the powers of $\mathrm{m}=t B_{Q}$, we have $t^{r} B_{Q} \subseteq A_{S}$ for some $r \in \mathbf{N}$. Define

$$
D=\prod_{j=1}^{r-1}(t \partial-j) \in \mathscr{D}\left(B_{Q}\right) \text {. }
$$

For any $n \in \mathbf{N}, D * t^{n}=\lambda_{n} t^{n}$ for some $\lambda_{n} \in k$, and further, $\lambda_{n}=0$ if and only if $1 \leqslant n \leqslant r-1$. Since $B_{Q}=k+k t+\ldots+k t^{r-1}+\mathfrak{m}^{r}$, this implies that $D * B_{Q}=$ $k+D * \mathfrak{m}^{r}$. For any $n \in \mathbf{N}$, and any $b \in B_{Q}$,

$$
(t \partial-j) *\left(t^{n} b\right)=t^{n}(t \partial-j+n) * b \in t^{n} B_{Q} .
$$

In particular, $D * \mathfrak{m}^{r} \subseteq \mathfrak{m}^{r}$, and hence $D * B_{Q} \subseteq k+\mathfrak{m}^{r} \subseteq A_{s}$. In other words,

$$
D \in \mathscr{D}\left(B_{S}, A_{S}\right)=A_{S} \otimes_{A} \mathscr{D}(B, A)
$$

by (2.7). Hence there exists $s \in S$ with $s D \in \mathscr{D}(B, A)=\mathscr{D}(\tilde{X}, X)$. In particular, as $D * 1 \in k \backslash\{0\}$, it follows that $s \in(s D) * B$. Consequently, $\mathscr{D}(\tilde{X}, X) * B$ is not contained in $M$.

Finally, since $M$ was an arbitrary maximal ideal of $\mathcal{O}(X)$ and $\mathscr{D}(\bar{X}, X) * \mathcal{O}(\bar{X})$ is a left $\mathscr{D}(X)$-submodule of $\mathscr{O}(X)$ (and hence an ideal), it follows that $\mathscr{D}(\tilde{X}, X) * \mathscr{O}(\tilde{X})=\mathscr{O}(X)$, as required.

Remarks. (1) As $X$ is affine, the category $\mathscr{D}(X)$-Mod is equivalent to $\mathscr{D}_{X}$-Mod, the category of quasi-coherent left $\mathscr{D}_{X}$-modules. Thus when $\pi: \bar{X} \rightarrow X$ is injective, the categories $\mathscr{D}_{\boldsymbol{X}}$-Mod and $\mathscr{D}_{\tilde{X}}$-Mod are equivalent.

(2) Essentially the same proof as above may be used to show that for any variety $X$ (not necessarily a curve), if $\tilde{X}$ is non-singular, and $\pi: \bar{X} \rightarrow X$ is injective, and \#Sing $X<\infty$ (that is, $X$ has only a finite number of singularities), then $\mathscr{D}(X)=\operatorname{End}_{\mathscr{D}(\bar{X})} \mathscr{D}(\bar{X}, X)$. However, if $\operatorname{dim} X>1$ then $\mathscr{D}(\bar{X}, X)$ will not be a projective $\mathscr{D}(\bar{X})$-module.

3.5. Corollary. Let $X$ be a curve, and suppose that $\pi: \tilde{X} \rightarrow X$ is injective. Then $\mathscr{D}(X)$ is a hereditary ring (that is, $\operatorname{gl} . \operatorname{dim} \mathscr{D}(X)=1$ ). 
Proof. By (1.4(e)), gl.dim $\mathscr{D}(\tilde{X})=1$, and the result follows because global dimension is a Morita invariant.

3.6. In order to prove the converse of Theorem 3.4 we require the following result which appears in [6, Theorem 1].

TheOREM. Let $X$ be a curve, and $x \in X$. Write $\overline{\mathcal{O}}_{X, x}$ for the integral closure of $\mathcal{O}_{X, x}$ in its field of fractions. Then $\pi$ is unramified at $\pi^{-1}(x)$ if and only if $\mathscr{D}\left(\mathscr{O}_{X, x}\right) \subseteq \mathscr{D}\left(\overline{\mathcal{O}}_{X, x}\right)$.

Corollary. Let $X$ be a curve and suppose that $\pi: \tilde{X} \rightarrow X$ is unramified at all points. Then $\mathscr{D}(X) \subseteq \mathscr{D}(\tilde{X})$.

Proof. Just observe that $\mathscr{D}(X)=\bigcap_{x \in \tilde{X}} \mathscr{D}_{X, \pi(x)}$ and $\mathscr{D}(\tilde{X})=\bigcap_{x \in \bar{X}} \mathscr{D}_{\tilde{X}, x}$; then apply the theorem.

3.7. It is an (almost) immediate corollary of (3.3) and (3.6) that if $X$ is a curve and $\pi: \tilde{X} \rightarrow X$ is unramified at some point in $\pi^{-1}$ (Sing $X$ ) then $\mathscr{D}(X)$ cannot be a simple ring. This observation can be extended to give the converse to Theorem 3.4.

THEOREM. Let $X$ be a curve and suppose that $\pi: \tilde{X} \rightarrow X$ is not injective. Then $\mathscr{D}(X)$ is not a simple ring, and consequently not Morita equivalent to $\mathscr{D}(\tilde{X})$.

Remark. Combined with Theorem 3.4 this proves the equivalence of Parts (a) and (b) of Theorem B of the introduction.

Proof. As before, write $A=\mathscr{O}(X)$ and $B=\mathscr{O}(\tilde{X})$. Since $\pi$ is not injective there exists a maximal ideal $\mathfrak{m}$ of $A$ and (at least) two distinct maximal ideals $\mathfrak{m}_{1}, \mathfrak{m}_{2}$ of $B$ which contain $\mathfrak{m}$. As $B$ is a Dedekind domain, $\mathfrak{m}_{1} \mathfrak{m}_{2}=\mathfrak{m}_{1} \cap \mathfrak{m}_{2}$. Thus $A \subseteq k+\mathfrak{m}_{1} \mathfrak{m}_{2} \subseteq B$. Let $Y$ be the curve with $\mathscr{O}(Y)=k+\mathfrak{m}_{1} \mathfrak{m}_{2}$. Then the morphism $\pi: \bar{X} \rightarrow Y$ is unramified at all points. By Corollary 3.6, it follows that $\mathscr{D}(Y) \subseteq \mathscr{D}(\tilde{X})$ (and these are distinct since $\mathscr{O}(Y) \neq \mathscr{O}(\tilde{X})$ ). Hence $\mathscr{D}(\bar{X}, Y)$, being a right ideal of $\mathscr{D}(\bar{X})$ and a left ideal of $\mathscr{D}(Y)$, is a proper two-sided ideal of $\mathscr{D}(Y)$. Since $\mathscr{D}(Y)$ is not simple, (3.3) shows that $1 \notin \mathscr{D}(\tilde{X}, Y) * \mathscr{O}(\bar{X})$. However, as $\mathscr{D}(\tilde{X}, X) \subseteq \mathscr{D}(\tilde{X}, Y)$, it follows that $1 \notin \mathscr{D}(\tilde{X}, X) * \mathscr{O}(\bar{X})$. By (3.3), we conclude that $\mathscr{D}(X)$ is not a simple ring.

3.8. An immediate question is whether $\mathscr{D}(X)$ and $\mathscr{D}(\tilde{X})$ being Morita equivalent actually implies that they are isomorphic. The answer is 'No', but there is some work involved. The easiest example to consider is the cusp $y^{2}=x^{3}$. Here $\tilde{X}=\mathbf{A}^{1}$ and if $\mathscr{O}(\tilde{X})=k[t]$, then $\mathscr{O}(X)$ may be identified with $k\left[t^{2}, t^{3}\right]$. Thus $\mathscr{D}(\bar{X})=k[t, \partial]$ (where $\partial=\partial / \partial t$ ), and one obtains

$$
\mathscr{D}(\tilde{X}, X)=t^{2} \mathscr{D}(\tilde{X})+(t \partial-1) \mathscr{D}(\tilde{X}) .
$$

Since $\pi: \tilde{X} \rightarrow X$ is injective, by (3.3) and (3.4), $\mathscr{D}(X)=\operatorname{End}_{\mathscr{D}(\bar{X})} \mathscr{D}(\tilde{X}, X)$. Fortunately, this ring has been computed explicitly in [19], and it is shown there that $\mathscr{D}(X)$ and $\mathscr{D}(\tilde{X})$ are not isomorphic (some changes in notation are required). 
More generally, it follows from [24] that $\mathscr{D}(X)$ and $\mathscr{D}(\tilde{X})$ are non-isomorphic whenever $X \neq \bar{X}=\mathbf{A}^{1}$.

Our interest in the questions in this paper was roused by a computation of Ian Musson. He computed $\mathscr{D}(X)$ when $X$ is the cusp $y^{2}=x^{3}$, and observed that $\mathscr{D}(X)$ was isomorphic to the endomorphism ring of the $\mathscr{D}(X)$-module

$$
t^{2} \mathscr{D}(\tilde{X})+(t \partial-1) \mathscr{D}(\tilde{X}),
$$

hence establishing the Morita equivalence of $\mathscr{D}(X)$ and $\mathscr{D}(\bar{X})$ for this specific example. This was an extremely surprising result (to us), and this paper is a consequence of our attempt to make this seem less of a surprise. We would like to thank Musson for sharing his results with us. He was also able to compute $\mathscr{D}(X)$ for various other monomial curves $X$, and in each case found a right-ideal of $\mathscr{D}\left(\mathbf{A}^{1}\right)$ such that $\mathscr{D}(X)$ was isomorphic to the endomorphism ring of that right ideal. See [17].

3.9. As remarked earlier, $\mathscr{D}(X)$ is filtered by its subspaces $\mathscr{D}^{n}(X)$ and the associated graded algebra gr $\mathscr{D}(X)=\bigoplus \mathscr{D}^{n}(X) / \mathscr{D}^{n-1}(X)$ is a commutative domain. When $X$ is a non-singular affine variety, then $\operatorname{gr} \mathscr{D}(X)$ is a finitely generated, noetherian $k$-algebra (1.4(a)), but for general $X$ it is an open problem as to exactly when gr $\mathscr{D}(X)$ has these properties. As an application of the earlier results of this section, we solve this question completely for $X$ a curve.

Some preliminary observations are required. As in (2.3) regard $\mathscr{D}(X)$ and $\mathscr{D}(\tilde{X})$ as subalgebras of $\mathscr{D}(K)$, where $K=$ Fract $\mathscr{O}(X)$. The order of $D \in \mathscr{D}(X)$ is the same whether $D$ is regarded as an element of $\mathscr{D}(X)$ or as an element of $\mathscr{D}(K)$. In particular,

$$
\text { gr } \mathscr{D}(X) \cong \bigoplus\left(\mathscr{D}^{n}(X)+\mathscr{D}^{n-1}(K) / \mathscr{D}^{n-1}(K)\right) \subseteq \operatorname{gr} \mathscr{D}(K) .
$$

We will always use this isomorphism to identify $\operatorname{gr} \mathscr{D}(X)$ and $\operatorname{gr} \mathscr{D}(\tilde{X})$ with subalgebras of $\mathrm{gr} \mathscr{D}(K)$.

3.10. We will need to compare the lattices of right ideals of $\mathscr{D}(\tilde{X})$ and gr $\mathscr{D}(\tilde{X})$. Let $I \subset J$ be distinct non-zero right ideals of $\mathscr{D}(\tilde{X})$. As $\operatorname{dim}_{k}(J / I)=\infty$, we have $\operatorname{dim}_{k}(\operatorname{gr} J / \operatorname{gr} I)=\infty$. Consequently, $\operatorname{gr} J / \operatorname{gr} I$ cannot be of finite length as a $\operatorname{gr} \mathscr{D}(\tilde{X})$-module. This leads one to consider the following dimension. Let $M \subseteq N$ be distinct non-zero ideals of $\operatorname{gr} \mathscr{D}(\bar{X})$. Then the 1-length of $N / M$ is defined to be the largest integer $n$ such that there exists a chain of ideals $M=M_{0} \subset M_{1} \subset \ldots \subset M_{n}=N$ such that $\operatorname{dim}_{k} M_{i} / M_{i-1}=\infty$ for each $i$. Since $\operatorname{gr} \mathscr{D}(\tilde{X})$ is a domain of Krull dimension 2, the 1-length of $N / M$ is always finite. The obvious induction therefore proves

(3.10.1) if $I \subset J$ are distinct, non-zero right ideals of $\mathscr{D}(\tilde{X})$ then

$$
\text { 1-length }(\text { gr } J / \text { gr } I) \geqslant \text { length }(J / I) \text {. }
$$

In fact, as we show next, there is often equality in (3.10.1). Let $\mathfrak{m}$ be a maximal ideal of $\mathcal{O}(\tilde{X})$. Because $\operatorname{gr} \mathscr{D}(\tilde{X})$ is isomorphic to the symmetric algebra over $\mathscr{O}(\tilde{X})$ of $\operatorname{Der}_{k} \mathcal{O}(\tilde{X})$, it follows that $\operatorname{gr}(\mathfrak{m} \mathscr{D}(\tilde{X}))=\mathfrak{m} \operatorname{gr} \mathscr{D}(\tilde{X})$ is a prime ideal of $\operatorname{gr} \mathscr{D}(\tilde{X})$. Of course, $\operatorname{gr}(\mathfrak{m} \mathscr{D}(\tilde{X}))$ is not a maximal ideal, and this implies that 
Since $\mathfrak{m} \mathscr{D}(\tilde{X})$ is a maximal right ideal of $\mathscr{D}(\tilde{X})$ by $(1.4(\mathrm{~g}))$, the obvious induction proves

(3.10.2) if $0 \neq a \in \mathscr{O}(\tilde{X})$ then

$$
\text { 1-length }(\operatorname{gr} \mathscr{D}(\tilde{X}) / \operatorname{gr}(a \mathscr{D}(\tilde{X})))=\text { length }(\mathscr{D}(\tilde{X}) / a \mathscr{D}(\tilde{X})) \text {. }
$$

Together (3.10.1) and (3.10.2) may be used to prove

Lemma. Let $J$ be a right ideal of $\mathscr{D}(\tilde{X})$ containing $0 \neq a \in \mathscr{O}(\tilde{X})$. Then

$$
\text { 1-length }(\operatorname{gr} \mathscr{D}(\tilde{X}) / \operatorname{gr} J)=\text { length }(\mathscr{D}(\tilde{X}) / J) \text {. }
$$

Proof. By (3.10.1),

$$
\text { 1-length }(\operatorname{gr} \mathscr{D}(\tilde{X}) / \operatorname{gr} J) \geqslant \text { length }(\mathscr{D}(\tilde{X}) / J)
$$

and

$$
\text { 1-length }(\operatorname{gr} J / \operatorname{gr}(a \mathscr{D}(\tilde{X}))) \geqslant \text { length }(J / a \mathscr{D}(\tilde{X})) \text {. }
$$

Because 1-length is additive on short exact sequences, combining this with (3.10.2) gives the result.

3.11. We prove the rather surprising result that $\operatorname{gr} \mathscr{D}(X)$ is isomorphic to a subalgebra of $\operatorname{gr} \mathscr{D}(\tilde{X})$, although $\mathscr{D}(X)$ is rarely a subalgebra of $\mathscr{D}(\tilde{X})$.

Proposition. Let $X$ be a curve. Then under the identification of (3.9), $\operatorname{gr} \mathscr{D}(X) \subseteq \operatorname{gr} \mathscr{D}(\tilde{X})$.

Proof. Choose $0 \neq a \in \mathscr{O}(\tilde{X})$ such that $a \mathscr{O}(\bar{X}) \subseteq \mathscr{O}(X)$. Then $a \in \mathscr{D}(\bar{X}, X)$ and thus $\mathscr{D}(X) a \subseteq \mathscr{D}(\tilde{X}, X) \subseteq \mathscr{D}(\tilde{X})$. Since $a \in \mathscr{D}^{0}(\tilde{X})$, this implies that

$$
\operatorname{gr} \mathscr{D}(X) \subseteq \operatorname{gr}\left(\mathscr{D}(\tilde{X}) a^{-1}\right)=\operatorname{gr} D(\tilde{X}) a^{-1} .
$$

Thus the subring $R=\operatorname{gr} \mathscr{D}(X) \operatorname{gr} \mathscr{D}(\tilde{X})$ of $\operatorname{gr} \mathscr{D}(K)$ is also contained in (gr $\mathscr{D}(\tilde{X})) a^{-1}$. But by $(1.4(\mathrm{e}))$, gr $\mathscr{D}(\bar{X})$ is a regular ring and hence integrally closed. Since $R \supseteq \operatorname{gr} \mathscr{D}(\tilde{X}) \supseteq R a$, this forces $R=\operatorname{gr} \mathscr{D}(\tilde{X})$. In particular, gr $\mathscr{D}(X) \subseteq \operatorname{gr} \mathscr{D}(\bar{X})$.

3.12. We can now determine precisely when $\mathscr{D}(X)$ is noetherian and/or finitely generated.

THEOREM. Let $X$ be a curve. Then the following are equivalent:

(a) $\operatorname{gr} \mathscr{D}(X)$ is a finitely generated $k$-algebra;

(b) $\operatorname{gr} \mathscr{D}(X)$ is noetherian;

(c) $\pi: \tilde{X} \rightarrow X$ is injective.

Remark. This proves the equivalence of Parts (a) and (d) of Theorem B in the introduction.

Proof. Suppose first that $\pi$ is not injective. The idea here is to mimic the well-known proof that $k+s k[s, t]$ is not noetherian. By (2.3) we have

$$
P=\mathscr{D}(\tilde{X}, X) \subseteq \mathscr{D}(X) \subseteq T=\operatorname{End}_{\mathscr{D}(\tilde{X})} P \subseteq \mathscr{D}(K),
$$


and by Theorem 3.7, $\mathscr{D}(X) \neq T$. Thus $J=P \mathscr{D}(X)$ is a two-sided ideal of $\mathscr{D}(X)$ which is a proper left ideal of $T$. Hence $\mathscr{D}(X) \subseteq \mathbf{I}_{T}(J)$ (notation (1.5)). However, $\mathbf{I}_{T}(J) / J$ is finite-dimensional over $k$, since $T$ and $\mathscr{D}(\tilde{X})$ are Morita equivalent (see $(1.4(\mathrm{~d}))$ and $(1.5))$. Hence $\operatorname{dim}_{k} \mathscr{D}(X) / J<\infty$.

Under the identifications of (3.9), $\operatorname{gr} J \subseteq \operatorname{gr} \mathscr{D}(X) \subseteq \operatorname{gr} T \subseteq \operatorname{gr} \mathscr{D}(K)$ and $\operatorname{gr} J$ is an ideal of gr $T$. However, $\operatorname{dim}_{k} T / J=\infty$ since $T$ is a simple ring (of infinite dimension), and hence $\operatorname{dim}_{k}(\operatorname{gr} T / \operatorname{gr} J)=\infty$. On the other hand, $\operatorname{dim}_{k}(\operatorname{gr} \mathscr{D}(X) / \mathrm{gr} J)<\infty$, so gr $T$ cannot be a finitely generated $\mathrm{gr} \mathscr{D}(X)$-module. However, for any $0 \neq a \in \operatorname{gr} J$, we have that $\operatorname{gr} T$ is isomorphic to the ideal $a(\operatorname{gr} T)$ of $\operatorname{gr} \mathscr{D}(X)$. Thus $a(\operatorname{gr} T)$ is a non-finitely generated ideal of $\operatorname{gr} \mathscr{D}(X)$. Whence gr $\mathscr{D}(X)$ is not noetherian, and consequently not a finitely generated $k$-algebra.

Now suppose that $\pi: \tilde{X} \rightarrow X$ is injective. As before write $P=\mathscr{D}(\tilde{X}, X)$ and set

$$
P^{*}=\{q \in \mathscr{D}(K) \mid q P \subseteq \mathscr{D}(\tilde{X})\} \cong \operatorname{Hom}_{\mathscr{D}(\bar{X})}(P, \mathscr{D}(\tilde{X})) .
$$

Clearly $P P^{*} \subseteq \operatorname{End}_{\mathscr{D}(\tilde{X})} P$, and so by Theorem $3.4, P P^{*} \subseteq \mathscr{D}(X)$. Note that this implies that $(\operatorname{gr} P)\left(\operatorname{gr} P^{*}\right) \subseteq \mathrm{gr} \mathscr{D}(X)$. Thus by (3.11) we obtain

$$
(\operatorname{gr} P)\left(\operatorname{gr} P^{*}\right) \subseteq \operatorname{gr} \mathscr{D}(X) \subseteq \operatorname{gr} \mathscr{D}(\tilde{X}) .
$$

The aim of the proof is to use (3.10) to show that

$$
\operatorname{dim}_{k} \operatorname{gr} \mathscr{D}(X) /(\operatorname{gr} P)\left(\operatorname{gr} P^{*}\right)<\infty,
$$

and then an appeal to Eakin's Theorem [14, §35] completes the proof.

Choose $0 \neq a \in \mathscr{O}(X)$ such that $a \mathscr{O}(\tilde{X}) \subseteq \mathscr{O}(X)$. Thus $a \mathscr{D}(\bar{X}) \subseteq P$ and by Lemma 3.10 ,

$$
\text { 1-length }(\text { gr } \mathscr{D}(\tilde{X}) / \text { gr } P)=\text { length }(\mathscr{D}(\bar{X}) / P) \text {. }
$$

Similarly, $P^{*} \subseteq \mathscr{D}(\bar{X}) a^{-1}$ and Lemma 3.10 may be applied to the left ideals $\mathscr{D}(\bar{X}) a \subseteq \mathscr{D}(\tilde{X})$ to prove that

$$
\text { 1-length }\left(\mathrm{gr} P^{*} / \operatorname{gr} \mathscr{D}(\tilde{X})\right)=\text { length }\left(P^{*} / \mathscr{D}(\tilde{X})\right) \text {. }
$$

However, since $\mathscr{D}(\tilde{X})$ is a hereditary domain, $\operatorname{Hom}_{\mathscr{D}(\tilde{X})}(-, \mathscr{D}(\tilde{X}))$ provides an order-reversing isomorphism between the lattice of submodules of $\mathscr{D}(\bar{X}) / P$ and that of $P^{*} / \mathscr{D}(\tilde{X})$. Thus length $(\mathscr{D}(\tilde{X}) / P)=\operatorname{length}\left(P^{*} / \mathscr{D}(\tilde{X})\right)$. Combining these three equalities gives

(3.12.2) 1-length $(\operatorname{gr} \mathscr{D}(\tilde{X}) / \operatorname{gr} P)=1$-length $\left(\operatorname{gr} P^{*} / \operatorname{gr} \mathscr{D}(\tilde{X})\right)=m$, say.

Now, observe that if $I_{1} \subseteq I_{2}$ and $J$ are non-zero ideals of $\operatorname{gr} \mathscr{D}(\bar{X})$ such that $\operatorname{dim}_{k}\left(I_{2} / I_{1}\right)=\infty$, then $\operatorname{dim}_{k}\left(J I_{2} / J I_{1}\right)=\infty$ also. Thus, if

$$
\operatorname{gr} \mathscr{D}(\bar{X})=I_{0} \subset I_{1} \subset \ldots \subset I_{m}=\operatorname{gr} P^{*}
$$

is a maximal chain for which $\operatorname{dim}_{k}\left(I_{j} / I_{j-1}\right)=\infty$ for all $j$, then

$$
\text { gr } P=(\operatorname{gr} P) I_{0} \subset \ldots \subset(\operatorname{gr} P) I_{m}=(\operatorname{gr} P)\left(\operatorname{gr} P^{*}\right)
$$

is also a chain in which each factor is infinite-dimensional. By (3.12.2) this is only possible if $\operatorname{dim}_{k}\left(\operatorname{gr} \mathscr{D}(\bar{X}) /(\operatorname{gr} P)\left(\operatorname{gr} P^{*}\right)\right)<\infty$. Thus by (3.12.1), $\operatorname{dim}_{k}(\operatorname{gr} \mathscr{D}(\tilde{X}) / \operatorname{gr} \mathscr{D}(X))<\infty$. Finally, by $(1.4(\mathrm{a})), \operatorname{gr} \mathscr{D}(\tilde{X})$ is a finitely generated $k$-algebra, and hence Eakin's Theorem implies that $\operatorname{gr} \mathscr{D}(X)$ is also a finitely generated $k$-algebra (and hence noetherian). 
Remarks. (1) It is shown in [5] and [26] that if $R$ is the algebra of germs of differential operators (with analytic coefficients) at an irreducible point of an analytic curve, then gr $R$ is noetherian (and generated by the analytic functions together with a finite number of differential operators). This corresponds to the case of $\pi$ being injective in the above theorem. The proofs in [5] and [26] are much more computational than the above.

(2) The dichotomy displayed in the above theorem can be well illustrated by two easy examples. In both examples $\tilde{X}=\mathbf{A}^{1}$. We write $\mathcal{O}(\tilde{X})=k[t]$ and $\partial=\partial / \partial t$.

First take $X$ to be the cusp $y^{2}=x^{3}$. Thus $\mathcal{O}(X)=k\left[t^{2}, t^{3}\right]$ and generators for $\mathscr{D}(X)$ are given in [5] (or [22] combined with (3.8)). They are

$$
t^{2}, t^{3}, t \partial, t^{2} \partial, \partial^{2}-2 t^{-1} \partial, t \partial^{2}-\partial, \partial^{3}-3 t^{-1} \partial^{2}+3 t^{-2} \partial
$$

(the final operator is superfluous for generating $\mathscr{D}(X)$ but is needed to write down generators for $\operatorname{gr} \mathscr{D}(X))$. Thus, viewing gr $\mathscr{D}(X) \subseteq \operatorname{gr} \mathscr{D}(\tilde{X})=k[t, s]$, we have

$$
\operatorname{gr} \mathscr{D}(X)=k\left[t^{2}, t^{3}, t s, t^{2} s, s^{2}, t s^{2}, s^{3}\right]=k+(t, s)^{2} .
$$

In contrast, if $X$ is the plane nodal curve $y^{2}=x^{2}(x+1)$ then

$$
\mathcal{O}(X) \cong k+\left(t^{2}-1\right) k[t],
$$

under the identification $x=t^{2}-1, y=t\left(t^{2}-1\right)$. It follows from Proposition 4.4 below, that $\mathscr{D}(X)=k+\left(t^{2}-1\right) \mathscr{D}(X)$. Consequently,

$$
\operatorname{gr} \mathscr{D}(X)=k+\left(t^{2}-1\right) k[t, s] \text {. }
$$

3.13. So far, in $\S \S 2$ and 3 we have given global information about $\mathscr{D}_{X}$, but it is also possible to obtain local information about the stalks $\mathscr{D}_{X, x}$. This information (all of which is implicit, if not explicit in the foregoing proofs) is summarised in the following.

Theorem. Let $X$ be a curve and $x \in X$. Then

(a) $\mathscr{D}_{X, x}$ is (right and left) noetherian;

(b) $\mathscr{D}_{X, x}$ is generated by $\mathcal{O}_{X, x}$ and a finite number of differential operators;

(c) $\mathscr{D}_{X, x}$ is simple if and only if $\# \pi^{-1}(x)=1$;

(d) if $\# \pi^{-1}(x)=1$, then $\mathscr{D}_{X, x}$ is Morita equivalent to $\mathscr{D}_{\bar{X}, \pi^{-1}(x)}$;

(e) gr $\mathscr{D}_{X, x}$ is noetherian (equivalently, gr $\mathscr{D}_{X, x}$ is a finitely generated $\mathscr{O}_{X, x^{-}}$ algebra) if and only if $\# \pi^{-1}(x)=1$.

3.14. Let $X$ be a curve, write $A=\mathscr{O}(X)$ and $B=\mathscr{O}(\tilde{X})$. As usual, set $P=\mathscr{D}(\tilde{X}, X)$ and $P^{*}=\{q \in Q \mid q P \subseteq \mathscr{D}(\tilde{X})\}$. Define

$$
\mathscr{D}(X, \tilde{X})=\{D \in \mathscr{D}(K) \mid D * \mathscr{O}(X) \subseteq \mathscr{O}(\tilde{X})\} .
$$

When $\mathscr{D}(X)$ and $\mathscr{D}(\tilde{X})$ are Morita equivalent, the equivalence of categories is implemented by the functor

$$
P \otimes_{\mathscr{D}(\tilde{X})}-: \mathscr{D}(\bar{X})-\operatorname{Mod} \rightarrow \mathscr{D}(X)-\operatorname{Mod} .
$$

The inverse functor is

$$
P^{*} \otimes_{\mathscr{D}(X)}-: \mathscr{D}(X)-\operatorname{Mod} \rightarrow \mathscr{D}(\tilde{X}) \text {-Mod, }
$$

and the next result gives a concrete description of the $\mathscr{D}(\tilde{X})-\mathscr{D}(X)$ bimodule $P^{*}$. 
Proposition. Let $X$ be a curve and suppose that $\mathscr{D}(X)$ and $\mathscr{D}(\tilde{X})$ are Morita equivalent. Then

$$
P^{*}=\mathscr{D}(X, \tilde{X}) \cong \mathscr{D}_{A}(A, B) .
$$

Proof. Note that $P^{*} \subseteq \mathscr{D}(K)$ because $P$ contains the conductor of $A$ in $B$. If $q \in \mathscr{D}(K)$, then $q \in P^{*}$ if and only if $q \mathscr{D}(\tilde{X}, X) * \mathscr{O}(\tilde{X}) \subseteq \mathscr{O}(X)$. Because $\mathscr{D}(X)$ and $\mathscr{D}(\bar{X})$ are Morita equivalent, Proposition 3.3 ensures that this condition is equivalent to $q * \mathscr{O}(X) \subseteq \mathcal{O}(\tilde{X})$. Hence $P^{*}=\mathscr{D}(X, \bar{X})$.

The second part of the proposition follows from the embedding $\mathscr{D}_{A}(A, B) \rightarrow$ $K \otimes_{A} \mathscr{D}_{A}(A, B) \cong \mathscr{D}(K)$. It is immediately clear that the image is precisely $\mathscr{D}(X, \tilde{X})$.

\section{Curves and finite-dimensional algebras}

4.1. Throughout this section $X$ will denote a curve. Theorem 3.4 may be considered as saying that, when $\pi$ is injective then the module and ideal structure of $\mathscr{D}(X)$ is completely determined by that of $\mathscr{D}(\tilde{X})$. One would therefore like to understand the structure of $\mathscr{D}(X)$ and its modules when $\pi$ is not injective (and hence when $\mathscr{D}(X)$ is not a simple ring). The aim of this section is to make progress in this direction.

Obvious test questions are to determine the two-sided ideal structure of $\mathscr{D}(X)$, to determine its global homological dimension, and to describe $\mathscr{D}(X)$ in terms of $\mathscr{D}(Y)$ for a 'better' curve $Y$. Partial answers to these questions are given. For the final question we have in mind a curve $Y$ for which there exist morphisms $\tilde{X} \stackrel{\psi}{\longrightarrow} Y \stackrel{\varphi}{\longrightarrow} X$ with $\psi$ unramified at all points, $\varphi$ injective, and $\varphi \psi=\pi$. The idea is to compare $\mathscr{D}(X)$ with $\mathscr{D}(Y)$, and $\mathscr{D}(Y)$ with $\mathscr{D}(\tilde{X})$.

4.2. After Theorem 2.5, the two-sided ideal structure of $\mathscr{D}(X)$ cannot be too complicated as the next result shows. Throughout this section we set $P=$ $\mathscr{D}(\tilde{X}, X), \quad T=\operatorname{End}_{\mathscr{D}(\tilde{X})} P, \quad J(X)=\operatorname{Ann}_{\mathscr{O}(X)}(\mathcal{O}(X) / P * \mathscr{O}(\tilde{X})), \quad$ and $\quad H(X)=$ $\mathscr{D}(X) / J(X)$.

Proposition. There is a unique minimal, non-zero two-sided ideal in $\mathscr{D}(X)$, namely $J(X)$. In particular, $J(X)^{2}=J(X)$ and $J(X)=P \mathscr{D}(X)$. The quotient $H(X)=\mathscr{D}(X) / J(X)$ is a finite-dimensional k-algebra. Furthermore, $J(X)$ is projective as a right (and as a left) $\mathscr{D}(X)$-module.

Proof. Write $J=J(X)$. The following inclusions are clear:

$$
T J * \mathcal{O}(X) \subseteq T *(P * \mathscr{O}(\tilde{X}))=T P * \mathscr{O}(\tilde{X})=P * \mathscr{O}(\tilde{X}) \subseteq \mathcal{O}(X) .
$$

Thus $T J \subseteq \mathscr{D}(X)$ and hence $T J \subseteq J$. In particular, $J$ is a left ideal of $T$. If $J^{\prime}$ is any non-zero two-sided ideal of $\mathscr{D}(X)$, then $J^{\prime}$ contains $J J^{\prime} J=(T J) J^{\prime}(T J)=J$ as $T$ is a simple ring. To see that $J \neq 0$ just observe that $0 \neq P \subseteq J$. Since $P \subseteq J(X)$ and $J(X)$ is the minimal non-zero ideal of $\mathscr{D}(X)$, certainly $J(X)=P \mathscr{D}(X)$. Clearly, $H(X)$ is finite-dimensional since $\mathcal{O}(X) / P * \mathscr{O}(\bar{X})$ is a finite-dimensional faithful $H(X)$-module.

To see that $J(X)$ is projective as claimed consider

$$
Q=\{q \in \mathscr{D}(X) \mid T q \subseteq \mathscr{D}(X)\}
$$


By the previous paragraph, $Q \supseteq J$. It is clear that $Q$ is a two-sided ideal of $\mathscr{D}(X)$ and a left ideal of $T$. Using the fact that $T$ is a simple ring we see that the following inclusions hold: $J \supseteq Q J Q=(T Q) J(T Q)=Q$. Hence $J=Q$. As $T$ is a simple ring, $J T=T$. By the Dual Basis Lemma, it follows that both $J_{\mathscr{D}(X)}$ and $\mathscr{D}(X) T$ are projective. The Morita equivalence of $T$ and $\mathscr{D}(\tilde{X})$ ensures that gl.dim $T=1$, and hence ${ }_{T} J$ is a projective. Thus ${ }_{\mathscr{D}(X)} J$ is projective.

Remarks. (1) Thus $\mathscr{D}(X)$ has only finitely many prime ideals, and the descending chain condition on ideals. There does exist an example where $\mathscr{D}(X)$ has infinitely many ideals (see $\S 5$ ).

(2) A natural question is to determine the precise structure of $H(X)$ and to determine how that structure depends on the nature of the singularities of $X$. For example, (3.4) and (3.7) tell us that $H(X)=0$ if and only if $\pi: \tilde{X} \rightarrow X$ is injective. Also in (4.13) we show that the structure of $H(X)$ is a local question. More precisely, $H(X) \cong \bigoplus H_{x}$, a direct sum of algebras $H_{x}$, one for each $x \in \operatorname{Sing} X$, and each $H_{x}$ depends only on the local ring $\mathcal{O}_{X, x}$.

(3) It is implicit in the Proposition that $\mathscr{D}(X)$ is simple if and only if $\mathscr{O}(X)$ is a simple $\mathscr{D}(X)$-module.

4.3. The following lemma is required in the proof of Proposition 4.4. It may be well known but does not appear in the literature.

Lemma. Let $Z$ be a non-singular variety. Let $A$ denote any localisation of $\mathcal{O}(Z)$, and let $I$ and $J$ be ideals of $A$. Suppose that

$$
\operatorname{Hom}_{\mathscr{D}(A)}(\mathscr{D}(A) / I \mathscr{D}(A), \mathscr{D}(A) / J \mathscr{D}(A)) \neq 0 .
$$

Then $\operatorname{Hom}_{A}(A / I, A / J) \neq 0$.

Proof. The hypothesis ensures that there exists $D \in \mathscr{D}(A)$ such that $D \notin J \mathscr{D}(A)$ and $D I \subseteq J \mathscr{D}(A)$. Hence $(D I) * A \subseteq J \mathscr{D}(A) * A=J$. But $D I * A=D * I$, and hence $D * I \subseteq J$. Thus $D: A \rightarrow A$ induces a differential operator $E: A / I \rightarrow A / J$. By $(1.3(\mathrm{e})), \mathscr{D}(A, J)=J \mathscr{D}(A)$ and so $D * A \subseteq J$. Hence $0 \neq E \in \mathscr{D}(A / I, A / J)$, and it follows from (1.3(a)) that $\operatorname{Hom}_{A}(A / I, A / J) \neq 0$.

Corollary. Let $A$ be as in the Lemma. Let $\mathrm{m}_{1}$ and $\mathrm{m}_{2}$ be distinct maximal ideals of $A$. Then

$$
\operatorname{Hom}_{\mathscr{D}(A)}\left(\mathscr{D}(A) / \mathfrak{m}_{1} \mathscr{D}(A), \mathscr{D}(A) / \mathfrak{m}_{2} \mathscr{D}(A)\right)=0 .
$$

4.4. Proposition. Suppose that the conductor, $I$, of $\mathscr{O}(X)$ in $\mathscr{O}(\tilde{X})$ is a product of (at least two) distinct maximal ideals of $\mathcal{O}(\bar{X})$, each occurring with multiplicity one. Then

(a) $J(X)=\mathscr{D}(\tilde{X}, X)=I \mathscr{D}(\tilde{X})$,

(b) $\mathscr{D}(X)=\mathscr{O}(X)+I \mathscr{D}(\tilde{X})$,

(c) $H(X) \cong \mathscr{O}(X) / I$.

Proof. Write $A=\mathscr{O}(X)$ and $B=\mathscr{O}(\tilde{X})$. First we show that $\pi: \bar{X} \rightarrow X$ is unramified at all points. It is enough to do it for points in $\pi^{-1}(\operatorname{Sing} X)$. For such a point the corresponding maximal ideal $\mathfrak{m}$ in $B$ contains $I$. Thus $\mathfrak{m}$ occurs in the 
expression of $I$ as a product of maximal ideals of $B$, and by hypothesis the power of $\mathfrak{m}$ occurring is 1 . Hence $\mathfrak{m} B_{\mathrm{m}}=I B_{\mathrm{m}}$ and $\pi$ is unramified at this point.

Since $\pi$ is unramified at all points, $\mathscr{D}(X) \subseteq \mathscr{D}(\tilde{X})$ by Corollary 3.6. Thus $P=\mathscr{D}(\tilde{X}, X) \subseteq \mathscr{D}(X) \subseteq \mathscr{D}(\tilde{X})$, and in particular $P$ is a two-sided ideal of $\mathscr{D}(X)$, and hence equal to $J(X)$ by (4.2). Notice that $I \subseteq P$. If

$$
I=\mathfrak{m}_{1} \ldots \mathfrak{m}_{n}=\bigcap\left\{\mathfrak{m}_{j} \mid 1 \leqslant j \leqslant n\right\}
$$

(a product of distinct maximal ideals of $B$ ), then

$$
\mathscr{D}(\tilde{X}) / 1 \mathscr{D}(\tilde{X}) \cong \bigoplus_{j=1}^{n} \mathscr{D}(\tilde{X}) / \mathfrak{m}_{j} \mathscr{D}(\tilde{X})
$$

Furthermore, the summands $\mathscr{D}(\bar{X}) / \mathfrak{m}_{j} \mathscr{D}(\tilde{X})$ are mutually non-isomorphic simple modules by $(1.4(\mathrm{~g}))$ and Corollary 4.3 . Consequently, any right ideal of $\mathscr{D}(\tilde{X})$ containing $I \mathscr{D}(\tilde{X})$ (in particular, $P)$ must be of the form $\left(\prod_{\alpha} \mathfrak{m}_{\alpha}\right) \mathscr{D}(\tilde{X})$, where the product is taken over some subset $\{\alpha\} \subseteq\{1, \ldots, n\}$. However,

$$
\left(\prod_{\alpha} \mathfrak{m}_{\alpha}\right) \mathscr{D}(\tilde{X}) * \mathscr{O}(\tilde{X})=\prod_{\alpha} \mathfrak{m}_{\alpha}
$$

which is not contained in $\mathscr{O}(X)$ unless $\{\alpha\}=\{1, \ldots, n\}$. Hence $P=I \mathscr{D}(\tilde{X})$. This proves (a).

Since $I$ is an ideal of $\mathcal{O}(\tilde{X})$, we have $\mathscr{O}(\tilde{X}) \subseteq \mathbf{I}_{\mathscr{D}(\bar{X})}(P)$ (notation (1.5)). As $\mathscr{D}(\tilde{X}) / P$ is a direct sum of $n=\operatorname{dim}_{k} \mathscr{O}(\tilde{X}) / I$ mutually non-isomorphic simple $\mathscr{D}(\tilde{X})$-modules, one has $\operatorname{End}_{\mathscr{D}(\tilde{X})}(\mathscr{D}(\tilde{X}) / P) \cong k \times \ldots \times k$ ( $n$ copies). Hence $\operatorname{dim}_{k}(\mathbf{I}(P) / P)=n$. However, $B+I \mathscr{D}(\tilde{X}) / I \mathscr{D}(\tilde{X}) \cong B / B \cap I \mathscr{D}(\tilde{X})=B / I$ is also of dimension $n$. Thus $\mathbf{I}(P)=\mathscr{O}(\tilde{X})+I \mathscr{D}(\tilde{X})$.

Finally, $\mathscr{D}(X) \subseteq \mathbf{I}(P)=\mathscr{O}(\tilde{X})+I \mathscr{D}(\tilde{X})$. Suppose that $D=f+D^{\prime} \in \mathscr{D}(X)$ for some $f \in \mathscr{O}(X), \quad D^{\prime} \in I \mathscr{D}(\tilde{X})$. Then $D * 1=f+D^{\prime} * 1 \in \mathscr{O}(X)$. In particular, $f \in \mathscr{O}(X)$. Thus $\mathscr{D}(X) \subseteq \mathscr{O}(X)+I \mathscr{D}(\tilde{X})$. The reverse inclusion is trivial, and (b) follows.

To obtain (c), note that

$$
H(X)=\mathscr{D}(X) / I \mathscr{D}(\tilde{X}) \cong \mathscr{O}(X) / \mathcal{O}(X) \cap I \mathscr{D}(\tilde{X})=\mathscr{O}(X) / I .
$$

4.5. Corollary. Suppose that the conductor, $I$, of $\mathcal{O}(X)$ in $\mathcal{O}(\bar{X})$ is a product of (at least two) distinct maximal ideals of $\mathcal{O}(\tilde{X})$ each occurring with multiplicity one. Then gl.dim $\mathscr{D}(X)=2$.

Proof. By hypothesis, $\mathcal{O}(X) / I$ is isomorphic to a product of copies of $k$. By the previous proposition $H(X) \cong \mathscr{O}(X) / I$, and hence $H(X)$ is semi-simple artinian. Hence by [8, Theorem 2.2], gl.dim $\mathscr{D}(X) \leqslant 2$. To show that $\operatorname{gl} . \operatorname{dim} \mathscr{D}(X) \neq 1$, we show that $\mathscr{D}(X)$ has a non-projective right ideal.

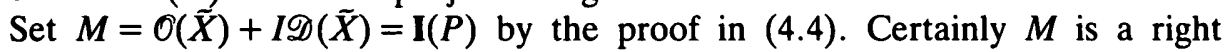
$\mathscr{D}(X)$-module properly containing $\mathscr{D}(X)$. For any $a \in I, a M \subseteq \mathscr{D}(X)$, and so $M$ is isomorphic to a right ideal of $\mathscr{D}(X)$. It therefore suffices to show that $M$ is not projective. Write

$$
M^{*}=\{x \in \mathscr{D}(X) \mid x M \subseteq \mathscr{D}(X)\} \cong \operatorname{Hom}_{\mathscr{D}(X)}(M, \mathscr{D}(X)) .
$$

By the Dual Basis Lemma [1, p. 203] it is enough to show that $1 \notin M M^{*}$. If $y \in M^{*}$ then certainly $y \mathscr{O}(\tilde{X}) \subseteq \mathscr{D}(X)$, and thus $y \mathscr{O}(\tilde{X}) * \mathscr{O}(X) \subseteq \mathscr{O}(X)$. But 


$$
\begin{gathered}
\mathscr{O}(\tilde{X}) * \mathscr{O}(X)=\mathscr{O}(\tilde{X}) ; \text { hence } y * \mathscr{O}(\tilde{X}) \subseteq \mathscr{O}(X) \text { and } y \in \mathscr{D}(\tilde{X}, X)=I \mathscr{D}(\tilde{X}) \text {. So } \\
M M^{*} \subseteq M I \mathscr{D}(\tilde{X})=I \mathscr{D}(\tilde{X}) \neq \mathscr{D}(X) .
\end{gathered}
$$

Remark. In fact one has $M^{*}=I \mathscr{D}(\tilde{X})=J(X)$, and by $(4.2), M^{*}$ is a projective left (and right) $\mathscr{D}(X)$-module.

4.6. Given a curve $X$ with normalization $\bar{X}$, we explain how to obtain a curve $Y$ and morphisms $\tilde{X} \stackrel{\psi}{\longrightarrow} Y \stackrel{\varphi}{\longrightarrow} X$ with $\varphi \psi=\pi$, such that $\varphi$ is injective and $\psi$ is unramified at all points. We follow the construction of curves outlined in [21, Chapitre IV, § 1]. From the description of $\mathscr{O}(Y)$ which will be obtained it will be possible to apply (4.4), (4.5) to obtain a good description of $\mathscr{D}(Y)$, while the proof of (3.4) may be imitated to describe $\mathscr{D}(X)$ in terms of $\mathscr{D}(Y)$.

For each $x \in X$, let $I_{x}$ be the ideal of functions in $\mathscr{O}(\tilde{X})$ which vanish on $\pi^{-1}(x)$. Then $k+I_{x}$ consists of those regular functions on $X$ which are constant on $\pi^{-1}(x)$. Set $R=\bigcap\left\{k+I_{x} \mid x \in X\right\}$. Since a regular function on $X$, when viewed as a function on $\tilde{X}$, is constant on each fibre $\pi^{-1}(x)$, one has $\mathcal{O}(X) \subseteq R \subseteq \mathcal{O}(\tilde{X})$. Thus $R$ is the co-ordinate ring of some curve, $Y$ say, with morphisms $\tilde{X} \stackrel{\psi}{\longrightarrow} Y \stackrel{\varphi}{\longrightarrow} X$ satisfying $\varphi \psi=\pi$. Let $z, z^{\prime} \in \tilde{X}$ belong to the same fibre $\pi^{-1}(x)$, with corresponding maximal ideals $\mathfrak{m}$ and $\mathfrak{m}^{\prime}$. As $I_{x} \subseteq \mathfrak{m}$, one has

$$
I_{x} \cap R \subseteq \mathfrak{m} \cap R \text { and } \mathfrak{m} \cap R \subseteq \mathfrak{m} \cap\left(k+I_{x}\right)=I_{x} .
$$

Consequently, $\mathfrak{m} \cap R=I_{x} \cap R=\mathfrak{m}^{\prime} \cap R$. In particular, $\psi(z)=\psi\left(z^{\prime}\right)$. This ensures that $\varphi$ is injective. If $x \in X$ is a non-singular point then $k+I_{x}=\mathscr{O}(\tilde{X})$, so $R$ may be expressed as a finite intersection, $\mathscr{O}(Y)=\bigcap\left\{k+I_{x} \mid x \in \operatorname{Sing} X\right\}$. Considering $R$ as the regular functions on $\tilde{X}$ which are constant on each fibre $\pi^{-1}(x)$, the conductor of $\mathscr{O}(Y)$ will be the largest ideal of $\mathscr{O}(\tilde{X})$ which is zero on $\pi^{-1}(\operatorname{Sing} X)$. This ideal is precisely

$$
\bigcap\left\{I_{x} \mid x \in \operatorname{Sing} X\right\}=\prod\left\{\mathfrak{m}_{z} \mid z \in \pi^{-1}(\operatorname{Sing} X)\right\} .
$$

In particular, the conductor is a product of distinct maximal ideals, each occurring with multiplicity one. Hence, by the first paragraph of the proof of (4.4), $\psi$ is unramified.

The description of $Y$ is completed by describing the local rings $\mathscr{O}_{Y, y}$. Let $y \in Y$, and write $x=\varphi(y)$. By definition $\mathscr{O}_{Y, y}$ consists of those rational functions on $Y$ which are regular in a neighbourhood of $y$ in $Y$. But $O(Y)$ consists of those functions regular on $X$ and constant on each fibre $\pi^{-1}(w)$ for $w \in X$. Hence $\mathscr{O}_{Y, y}$ consists of those rational functions on $\tilde{X}$ which are regular on a neighbourhood of $\pi^{-1}(x)=\psi^{-1}(y)$, and constant on the fibre $\pi^{-1}(x)$ (the condition on constancy on the other fibres can be ignored by choosing a sufficiently small neighbourhood). Hence $\mathcal{O}_{Y, y}=k+\mathrm{r}_{y}$ where $\mathrm{r}_{y}$ is the Jacobson radical of the semi-local ring $\mathcal{O}_{\bar{X}, \pi^{-1}(x)}$, corresponding to $\pi^{-1}(x)=\psi^{-1}(y) \subseteq \bar{X}$.

We summarise the above in the following proposition.

Proposition. Let $Y$ be as above. Then $\pi: \tilde{X} \rightarrow X$ factorises through

$$
\tilde{X} \stackrel{\psi}{\longrightarrow} Y \stackrel{\varphi}{\longrightarrow} X
$$


with $\psi$ unramified at all points and $\varphi$ injective. For each $y \in Y, \mathscr{O}_{Y, y}=k+r_{y}$ where $\mathfrak{r}_{y}$ is the radical of the semi-local ring $\mathcal{O}_{\tilde{X}, \psi^{-1}(y)}$. Furthermore, the conductor of $\mathcal{O}(X)$ in $\mathcal{O}(Y)$ is a product of distinct maximal ideals of $\mathcal{O}(Y)$.

4.7. The next goal is to describe $\mathscr{D}(X)$ in terms of $\mathscr{D}(Y)$. We will prove (eventually) that $\mathscr{D}(Y, X) * \mathscr{O}(Y)=\mathscr{O}(X)$ and then apply Lemma 3.2. This equality will be established locally and the next two lemmas are required to obtain the local equality.

LEMmA. Let $B$ be a regular $k$-algebra of finite type with $\operatorname{dim} B=1$. Let $r \geqslant 2$, and let $\mathfrak{m}_{1}, \ldots, \mathfrak{m}_{r}$ be distinct maximal ideals of $B$. Write $I=\mathfrak{m}_{1}^{i_{1}} \ldots \mathfrak{m}_{r}^{i_{r}}$ (where each $\left.i_{j} \geqslant 1\right), C=k+I$, and $A=k+I_{1}$. Then $\mathscr{D}(C, A) * C=A$.

Proof. For each $j$, choose $t_{j} \in \mathfrak{m}_{j}$ such that $\mathfrak{m}_{j}=\mathfrak{m}_{j}^{2}+k t_{j}$. Write $x=t_{1} t_{2} \ldots t_{r}$, and $y=t_{1}^{i_{1}} \ldots t_{r}^{i_{r}}$. As $B$ is regular, $\operatorname{dim}_{k}\left(I / I \mathrm{~m}_{1}\right)=1$, and so $I=I \mathrm{~m}_{1}+k y$; thus $C=A+k y$.

As $B$ is regular, there exists $\partial \in \operatorname{Der}_{k} B$ such that $\partial * t_{1} \notin \mathfrak{m}_{1}$. For any derivation $\delta$, and any maximal ideal $\mathfrak{m}, \delta * \mathfrak{m}^{2} \subseteq \mathfrak{m}$. Hence the product rule implies that $(x \partial) *\left(I \mathfrak{m}_{1}\right) \subseteq I \mathfrak{m}_{1}$. Also $\left(t_{2} \ldots t_{r} \partial\right) * t_{1} \in B \backslash \mathfrak{m}_{1}$ and thus

$$
(x \partial) * y \in I \backslash I \mathrm{~m}_{1} \text {. }
$$

Thus $(x \partial) * y=\alpha y+h$ for some $\alpha \in k \backslash\{0\}$ and $h \in I \mathrm{~m}_{1}$. As $(x \partial) * 1=0$, these observations imply that $(\alpha-x \partial) * C \subseteq A$, and $(\alpha-x \partial) * 1=\alpha \neq 0$. Thus $1 \epsilon$ $\mathscr{D}(C, A) * C$, and $A=\mathscr{D}(C, A) * C$.

4.8. Lemma. Let $B$ be a regular $k$-algebra of finite type with $\operatorname{dim} B=1$. Let $r \geqslant 2$, and let $\mathfrak{m}_{1}, \ldots, \mathfrak{m}_{r}$ be distinct maximal ideals of $B$. Set $C=k+\mathfrak{m}_{1} \ldots \mathfrak{m}_{r}$ and $A=k+\mathfrak{m}_{1}^{i_{1}} \ldots \mathfrak{m}_{r}^{i_{r}}\left(\right.$ where each $\left.i_{j} \geqslant 1\right)$. Then $\mathscr{D}(C, A) * C=A$.

Proof. Use the previous lemma and induction.

4.9. Corollary. Let $B$ be a regular $k$-algebra of finite type with $\operatorname{dim} B=1$. Let $r \geqslant 2$, and let $\mathfrak{m}_{1}, \ldots, \mathfrak{m}_{r}$ be distinct maximal ideals of $B$. Set $C=k+\mathfrak{m}_{1} \ldots \mathfrak{m}_{r}$, and let $A \subseteq C$ be any subalgebra such that $\left(\mathrm{m}_{1} \ldots \mathrm{m}_{r}\right)^{n} \subseteq A$ for $n$ sufficiently large. Then $\mathscr{D}(C, A) * C=A$.

Proof. Just observe that if $A^{\prime}=k+\mathfrak{m}_{1}^{n} \ldots \mathfrak{m}_{r}^{n}$ then the previous lemma gives $\mathscr{D}\left(C, A^{\prime}\right) * C=A^{\prime}$. In particular, $1 \in \mathscr{D}\left(C, A^{\prime}\right) * C \subseteq \mathscr{D}(C, A) * C$. Therefore the result follows.

4.10. Proposition. Let $X$ and $Y$ be as in (4.6). Then $\mathscr{D}(Y, X) * \mathscr{O}(Y)=\mathscr{O}(X)$.

Proof. Write $Q=\mathscr{D}(Y, X)$. Since $Q * \mathscr{O}(Y)$ is an $\mathscr{O}(X)$-submodule of $\mathscr{O}(X)$ it is enough to prove the result locally. Pick $y \in Y$ and put $x=\varphi(y)$. Since $\varphi$ is injective $\mathcal{O}_{Y, y}=\mathscr{O}_{X, x} \otimes_{\mathcal{O}(X)} \mathcal{O}(Y)$. Thus

$$
\mathcal{O}_{X, x} \otimes_{\mathcal{O}(X)} Q=\mathscr{D}\left(\mathcal{O}_{X, x} \otimes_{\mathcal{O}(X)} \mathscr{O}(Y), \mathscr{O}_{X, x}\right)=\mathscr{D}\left(\mathcal{O}_{Y, y}, \mathcal{O}_{X, x}\right),
$$

where the first equality comes from (2.7).

By Proposition $4.6, \mathcal{O}_{Y, y}=k+\mathfrak{m}_{1} \ldots \mathfrak{m}_{r}$ where $\mathfrak{m}_{1}, \ldots, \mathfrak{m}_{r}$ are the maximal ideals of the semi-local ring $\mathcal{O}_{\bar{X}, \pi^{-1}(x)}=\mathscr{O}_{X, \psi^{-1}(y)}$. Applying Corollary 4.9 with 
$C=\mathscr{O}_{Y, y}$ and $A=\mathscr{O}_{X, x}$ shows that $\mathscr{D}\left(\mathcal{O}_{Y, y}, \mathscr{O}_{X, x}\right) * \mathscr{O}_{Y, y}=\mathscr{O}_{X, x}$. Thus the local equality of $\mathscr{D}(Y, X) * \mathscr{O}(Y)$ and $\mathscr{O}(X)$ is established, and the result follows.

4.11. Combining the earlier results of this section gives the following theorem. The reader is reminded that a right ideal $I$ of a ring $R$ is said to be generative if $R I=R$. Clearly such a right ideal is a generator in Mod- $R$.

THEOREM. Let $X$ and $Y$ be curves with morphisms $\tilde{X} \stackrel{\psi}{\longrightarrow} Y \stackrel{\varphi}{\longrightarrow} X$ such that $\varphi \psi=\pi, \psi$ is unramified at all points, and $\varphi$ is injective. Assume that $\pi$ is not injective. Then

(a) $\operatorname{gl} \cdot \operatorname{dim} \mathscr{D}(Y)=2$;

(b) $H(Y) \cong k \times \ldots \times k$ where there is one copy of $k$ for each point in Sing $Y$;

(c) $\mathscr{D}(X) \cong \operatorname{End}_{\mathscr{D}(Y)} \mathscr{D}(Y, X)$;

(d) $\mathscr{D}(Y, X)$ is a generative right ideal of $\mathscr{D}(Y)$, and a projective left ideal of $\mathscr{D}(X)$.

Remark. Given Part (d), it is natural to ask whether $\mathscr{D}(Y, X)$ is a projective $\mathscr{D}(Y)$-module, as this would imply that $\mathscr{D}(X)$ and $\mathscr{D}(Y)$ are Morita equivalent. Unfortunately, this is not so, as we show by an example in (5.8). Thus the ideal structure of $\mathscr{D}(X)$ may be more complicated than that of $\mathscr{D}(Y)$.

Proof. Parts (a) and (b) follow from (4.4) and (4.5) given the description of $Y$ in (4.6). Part (c) follows from (4.10) and (3.2). To see that $Q=\mathscr{D}(Y, X)$ is generative, it is enough to show that it is not contained in any proper two-sided ideal of $\mathscr{D}(Y)$. Suppose to the contrary that $M$ is a maximal (two-sided) ideal of $\mathscr{D}(Y)$ containing $Q$. By (4.2) and (4.4) such an $M$ is of the form $\mathscr{D}(\tilde{X}, Y)+\mathfrak{m}$ for some maximal ideal $\mathfrak{m}$ of $\mathscr{O}(Y)$. But now $\mathscr{D}(Y, X) * \mathscr{O}(Y) \subseteq M * \mathscr{O}(Y) \subseteq \mathfrak{m}$. This contradicts Proposition 4.10, and proves that $\mathscr{D}(Y, X)$ is generative. Finally, since $Q \mathscr{D}(Y) \subseteq Q \subseteq \mathscr{D}(X)$, certainly $\mathscr{D}(Y) \subseteq Q^{*}=\operatorname{Hom}_{\mathscr{D}(X)}(Q, \mathscr{D}(X))$. Thus $1 \in \mathscr{D}(Y) Q \subseteq Q^{*} Q$ and the Dual Basis Lemma implies that $Q$ is a projective left $\mathscr{D}(X)$-module.

4.12. Corollary. Let $X$ be a curve. Then $\operatorname{gl} \cdot \operatorname{dim} X=1$ if and only if $\pi: \tilde{X} \rightarrow X$ is injective.

Proof. Suppose that $\pi$ is not-injective and take $Y$ as in (4.6). Write $Q=\mathscr{D}(Y, X)$ and $R=\operatorname{End}_{\mathscr{D}(X)} Q$. Then $R \supseteq \mathscr{D}(Y)$. But, by (4.11(d)), $\mathscr{D}(Y) R=$ $\mathscr{D}(Y) Q R=\mathscr{D}(Y) Q=\mathscr{D}(Y)$. Thus $R=\mathscr{D}(Y)$. Thus, if $\operatorname{gl} \cdot \operatorname{dim} \mathscr{D}(X)=1$ then by [1, Ex. 22.17, p. 269], gl.dim $\mathscr{D}(Y)=1$ also. This contradicts Theorem 4.11(a). The converse is just Corollary 3.5.

Remark. This completes the proof of Theorem B of the Introduction.

4.13. To obtain a better description of $\mathscr{D}(X)$ it is necessary to be able to compute $H(X)$. This is a local problem as the next result shows.

Theorem. Let $X$ be a curve and $x \in X$. Then $\mathscr{D}_{X, x}$ has a unique minimal non-zero ideal, $J_{x}$ say, which satisfies

(a) $J_{x}=\mathscr{O}_{X, x} \otimes_{O(X)} J(X)=\mathscr{D}_{X, x} J(X)$;

(b) $J_{x}=\operatorname{Ann}_{\mathscr{D}_{X, X}}\left(\mathcal{O}_{X, x} / P_{x} * \mathscr{O}_{\tilde{X}, \pi^{-1}(x)}\right)$ where $\mathcal{O}_{\bar{X}, \pi^{-1}(x)}$ is the semi-local ring cor- 
responding to $\pi^{-1}(x) \subseteq \tilde{X}$, and

$$
P_{x}=\mathscr{D}\left(\mathcal{O}_{\tilde{X}, \pi^{-1}(x)}, \mathcal{O}_{X, x}\right)=\mathscr{O}_{X, x} \otimes_{\mathscr{O}(X)} \mathscr{D}(\tilde{X}, X) ;
$$

(c) $H_{x}:=\mathscr{D}_{X, x} / J_{x} \cong \mathscr{O}_{X, x} \otimes_{\mathcal{O}(X)} H(X)$ is a finite-dimensional $k$-algebra;

(d) $H_{x}=0$ if and only if $\# \pi^{-1}(x)=1$;

(e) $J(X)=\bigcap\left\{J_{x} \mid x \in \operatorname{Sing} X\right\}$;

(f) $H(X) \cong \bigoplus\left\{H_{x} \mid x \in \operatorname{Sing} X\right\}$.

Proof. To start, define $J_{x}$ as in (b). The same proof as in (4.2) then ensures that $J_{x}$ is the unique minimal non-zero ideal of $\mathscr{D}_{X, x}$, and that $\mathscr{D}_{X, x} / J_{x}$ is finitedimensional. Because $J_{x} \cap \mathscr{D}(X)$ is a non-zero ideal of $\mathscr{D}(X)$, it follows that $J(X) \subseteq J_{x}$. Thus $\mathscr{D}_{X, x} J(X) \cong \mathscr{O}_{X, x} \otimes_{\mathcal{O}(X)} J(X)$ is a two-sided ideal of $\mathscr{D}_{X, x}$ contained in $J_{x}$ and hence equal to $J_{x}$. This proves (a), and also gives the isomorphism in (c). The statement of (d) is just a local version of Theorem 3.4 (and, in fact, is part of the proof of that result). Since $J_{x}=\mathcal{O}_{X, x} \otimes_{O(X)} J(X)$, Part (e) is an immediate consequence of (d). It remains to prove (f). For any distinct $x, y \in \operatorname{Sing} X$, then $\mathfrak{m}_{x}^{n} \subseteq J_{x} \cap \mathscr{D}(X)$ for some integer $n$, and similarly for $y$. Thus $J_{x} \cap \mathscr{D}(X)+J_{y} \cap \mathscr{D}(X)=\mathscr{D}(X)$, and the Chinese Remainder Theorem implies that

$$
\mathscr{D}(X) / J(X) \cong \bigoplus\left\{\mathscr{D}(X) / J_{x} \cap \mathscr{D}(X) \mid x \in \operatorname{Sing} X\right\} .
$$

However, $\mathfrak{m}_{x}^{n} \subseteq J_{x} \cap \mathscr{D}(X)$ implies that

$$
\mathscr{D}(X) / J_{x} \cap \mathscr{D}(X) \cong \mathscr{D}(X)+J_{x} / J_{x} \cong \mathscr{O}_{X, x} \otimes_{\mathscr{O}(X)}\left(\mathscr{D}(X)+J_{x} / J_{x}\right)=\mathscr{D}_{X, x} / J_{x}=H_{x} .
$$

Remark. This result was prompted by A. Schofield asking whether (f) was true. We would like to thank him for his interest.

4.14. For a variety $X$, there is a natural decomposition $\mathscr{D}(X)=\mathscr{O}(X)+L_{X}$ where $L_{X}$ is defined to be the left ideal which is the annihilator of $1 \in \mathcal{O}(X)$; that is $L_{X}=\{D \in \mathscr{D}(X) \mid D * I=0\}$. In [2] the question is raised as to whether for different varieties $X$ and $X^{\prime}$ one can have $L_{X}=L_{X^{\prime}}$. The question can be thought of as asking whether distinct varieties have rings of differential operators that are different in more than just the obvious way (namely that the multiplication operators are different). An example is given in [2] showing that one can have $L_{X}=L_{X^{\prime}}$ for non-isomorphic $X$ and $X^{\prime}$. The example is based on the BernsteinGelfand-Gelfand example [3] and the varieties $X$ and $X^{\prime}$ are of dimension 2. Here we show that even for non-isomorphic curves $X$ and $X^{\prime}$ one can have $L_{X}=L_{X^{\prime}}$

Example. Set $B=k[t]=\mathcal{O}\left(\mathbf{A}^{1}\right)$, and set $x=t(t-1)(t-2)(t-3)$. Define curves $X$ and $X^{\prime}$, both of which have normalisation $\mathbf{A}^{1}$, by $\mathcal{O}(X)=k+x B$, and $\mathcal{O}\left(X^{\prime}\right)=k+k t(t-3)+x B$. Then

$$
\mathscr{D}(X)=\mathscr{O}(X) \bigoplus x \mathscr{D}\left(\mathbf{A}^{1}\right) \partial \text { and } \mathscr{D}\left(X^{\prime}\right)=\mathscr{O}\left(X^{\prime}\right) \oplus x \mathscr{D}\left(\mathbf{A}^{1}\right) \partial \text {, }
$$

where $\partial=\partial / \partial t$. In particular, $L_{X}=L_{X^{\prime}}$.

Proof. Note that $(t(t-3))^{2} \in k+k t(t-3)+x B$, so $\mathcal{O}\left(X^{\prime}\right)$ really is a ring. In both cases the conductor in $B$ is $x B$ which is a product of distinct maximal ideals 
of $B$. Hence by Proposition 4.4(b),

$$
\mathscr{D}(X)=\mathscr{O}(X)+x \mathscr{D}\left(\mathbf{A}^{1}\right)=\mathscr{O}(X) \bigoplus x \mathscr{D}\left(\mathbf{A}^{1}\right) \partial,
$$

and similarly

$$
\mathscr{D}\left(X^{\prime}\right)=\mathscr{O}\left(X^{\prime}\right) \oplus x \mathscr{D}\left(\mathbf{A}^{1}\right) \partial .
$$

4.15. Let $X$ be a non-singular curve, and $I$ a non-zero ideal of $\mathscr{O}(X)$. In general $\mathscr{O}(X) / I$ is not a direct sum of simple $\mathscr{O}(X)$-modules. However, as we show below, $\mathscr{D}(X) / I \mathscr{D}(X) \cong(\mathscr{O}(X) / I) \otimes_{O(X)} \mathscr{D}(X)$ is always a direct sum of simple $\mathscr{D}(X)$ modules. An amusing proof can be given by introducing a singular curve into the problem, and using the results in $\S 3$.

Proposition. Let $X$ be a non-singular curve, and I a non-zero ideal of $O(X)$. Suppose that $I=\mathfrak{m}_{1}^{i_{1}} \ldots \mathfrak{m}_{r}^{i_{r}}$ where the $\mathfrak{m}_{j}$ are distinct maximal ideals of $\mathcal{O}(X)$. Then $\mathscr{D}(X) / I \mathscr{D}(X) \cong \bigoplus_{j=1} M_{j}$ where, for each $j, M_{j}$ is a direct sum of $i_{j}$ copies of the simple $\mathscr{D}(X)$-module $\mathscr{D}(X) / \mathfrak{m}_{j} \mathscr{D}(X)$. Furthermore, if $j \neq l$ then

$$
\operatorname{Hom}_{\mathscr{D}(X)}\left(M_{j}, M_{l}\right)=0 .
$$

Proof. The crucial step in the proof is to show that if $m$ is a maximal ideal of $\mathcal{O}(X)$, then

$$
\mathscr{D}(X) / \mathfrak{m}^{2} \mathscr{D}(X) \cong \mathscr{D}(X) / \mathfrak{m} \mathscr{D}(X) \bigoplus \mathscr{D}(X) / \mathfrak{m} \mathscr{D}(X) .
$$

We begin by proving this. Define $A=k+\mathfrak{m}^{2}$ and let $X^{\prime}$ be the curve with $\mathcal{O}\left(X^{\prime}\right)=A$. The normalisation of $X^{\prime}$ is $X$ and the natural projection $\pi: X \rightarrow X^{\prime}$ is injective. Observe that $\mathfrak{m}^{2} \mathscr{D}(X) \subseteq \mathscr{D}\left(X, X^{\prime}\right) \subseteq \mathscr{D}(X)$. By (3.3) and (3.4), $\mathscr{D}\left(X, X^{\prime}\right) * \mathscr{O}(X)=\mathscr{O}\left(X^{\prime}\right)$, whence $\mathscr{D}\left(X, X^{\prime}\right)$ cannot equal either $\mathfrak{m}^{2} \mathscr{D}(X)$ or $\mathfrak{m} \mathscr{D}(X)$. Hence the module $\mathscr{D}(X) / \mathfrak{m}^{2} \mathscr{D}(X)$ which is of length 2 by $(1.4(\mathrm{~g}))$ is not uniserial. It therefore splits as required.

The result quickly follows. First write $\mathcal{O}(X) / I$ as a direct sum of its primary components and reduce to the case where $I=\mathfrak{m}^{n}$. Because $\mathscr{D}(X) / \mathfrak{m}^{2} \mathscr{D}(X)$ splits, by induction, $\mathscr{D}(X) / \mathfrak{m}^{n} \mathscr{D}(X)$ splits as a direct sum of copies of $\mathscr{D}(X) / \mathfrak{m} \mathscr{D}(X)$. Finally the fact that $\operatorname{Hom}_{\mathscr{D}(X)}\left(M_{j}, M_{l}\right)=0$ for $j \neq l$ follows from (4.3).

REMARK. It is certainly possible to give proofs of this result without introducing the singular curve $X^{\prime}$. One such, shown to us by R. B. Warfield (unpublished), rests on the fact that $\mathscr{D}(X) / \mathfrak{m} \mathscr{D}(X)$ is isomorphic, as an $\mathscr{O}(X)$-module, to the injective hull of $\mathcal{O}(X) / \mathrm{m}$. The Proposition is used in (5.6).

\section{Examples}

5.1. The results so far are not sufficient to give a precise description of the ideal structure of $\mathscr{D}(X)$ when $X$ is an arbitrary curve. The examples in this section illustrate various features of the structure of $\mathscr{D}(X)$. For example, we show that $\mathscr{D}(X)$ may have infinitely many ideals.

We leave as open questions (i) whether $\mathscr{D}(X)$ may have infinite, or arbitrarily large finite global homological dimension, and (ii) what restrictions are there on the structure of $H(X)$-can any finite-dimensional algebra occur? 
5.2. We begin with a fairly general situation. Let $X$ be a singular curve with a unique singularity, and assume that $\pi: \tilde{X} \rightarrow X$ is unramified. Write $A=\mathscr{O}(X)$ and $B=\mathcal{O}(\tilde{X})$. Let $\mathfrak{m}$ be the maximal ideal of $\mathcal{O}(X)$ corresponding to the singular point, and let $\left\{\mathfrak{m}_{\lambda} \mid \lambda \in \Lambda\right\}$ be the distinct maximal ideals of $B$ containing $\mathfrak{m}$. Then (i) $\mathfrak{m} B=\prod_{\lambda} \mathfrak{m}_{\lambda}$ (as $\pi$ is unramified), and (ii) the conductor $I=\operatorname{Ann}_{A}(B / A)$ is of the form $I=\prod_{\lambda} \mathfrak{m}_{\lambda}^{i_{\lambda}}$ with each $i_{\lambda} \geqslant 1$ (as $X$ has a unique singular point).

\subsection{Lemma (Notation (5.2)). $\mathscr{D}(B, A)=I \mathscr{D}(B)$.}

Proof. The argument is by induction on the $i_{\lambda}$. If all $i_{\lambda}=1$, then by (4.4) the lemma holds. Suppose for some $v \in \Lambda$ that $i_{v}>1$. Define $A^{\prime}=A+\Pi_{\lambda} \mathrm{m}_{\lambda}^{i_{\lambda}}$ where $j_{\lambda}=i_{\lambda}$ for $\lambda \neq v$ and $j_{v}=i_{v}-1$. Then $A \subseteq A^{\prime} \subseteq B$. Applying the induction hypothesis to $A^{\prime}=\mathscr{O}\left(X^{\prime}\right)$ with $I^{\prime}=\operatorname{Ann}_{A^{\prime}}\left(B / A^{\prime}\right)$, we have $\mathscr{D}\left(B, A^{\prime}\right)=I^{\prime} \mathscr{D}(B)$.

Now $I \mathscr{D}(B) \subseteq \mathscr{D}(B, A) \subseteq \mathscr{D}\left(B, A^{\prime}\right)=I^{\prime} \mathscr{D}(B)$. However, $\mathfrak{m} I^{\prime} \subseteq I$ and $\mathfrak{m}$ is a product of distinct maximal ideals of $B$. Thus the argument used in the proof of (4.4) shows that $\mathscr{D}(B, A)$ must be of the form $I^{\prime \prime} \mathscr{D}(B)$ for some ideal $I^{\prime \prime}$ of $B$ satisfying $I \subset I^{\prime \prime} \subset I^{\prime}$. But $I^{\prime \prime} \mathscr{D}(B) * B=I^{\prime \prime}$ which forces $I^{\prime \prime}=I$. Hence the result follows.

5.4. Lemma. Let $X$ be a curve with $\pi: \tilde{X} \rightarrow X$ unramified at all points. Suppose there exists an ideal $I$ of $\mathscr{O}(X)$ such that $\mathscr{D}(\tilde{X}, X)=I \mathscr{D}(\tilde{X})$. Then the unique minimal ideal of $\mathscr{D}(X)$ is $J(X)=I \mathscr{D}(\tilde{X})=\mathscr{D}(\mathcal{O}(X), I)$.

Proof. By (3.6), $\mathscr{D}(X) \subseteq \mathscr{D}(\tilde{X})$, and hence $\mathscr{D}(\tilde{X}, X)$ becomes an ideal of $\mathscr{D}(X)$. Thus, by $(4.2), J(X)=\mathscr{D}(\tilde{X}, X)$. However, by hypothesis,

$$
\mathscr{D}(\tilde{X}, X) * \mathscr{O}(\bar{X})=I,
$$

and the description of $J(X)$ in (4.2) gives

$$
J(X)=\{D \in \mathscr{D}(X) \mid D * \mathscr{O}(X) \subseteq I\}=\mathscr{D}(\mathscr{O}(X), I) .
$$

But $I \mathscr{D}(\tilde{X})=\mathscr{D}(\mathscr{O}(\tilde{X}), I) \subseteq \mathscr{D}(\mathscr{O}(X), I)$ where the first equality comes from (1.3(e)). The lemma follows.

5.5. The problem of describing the two-sided ideal structure of $H(X)$ for $X$ as in (5.2) is thus reduced to describing the structure of $H(X)=\mathscr{D}(X) / J(X)=$ $\mathscr{D}(X) / I \mathscr{D}(\tilde{X})$. Note that $\mathscr{O}(X) / I$ is a faithful $H(X)$-module, so $H(X)$ embeds in $\operatorname{End}_{k}(\mathcal{O}(X) / I)$.

As an easy application of the foregoing consider the following example. Suppose that $B=k[t]$, let $x \in B$ be of degree at least 2, and suppose that $x$ is a product of distinct linear factors (that is, each root of $x$ occurs with multiplicity 1). Write $A=k+k x+x^{2} B$, set $\mathcal{O}(X)=A$ and note that $A$ satisfies the hypothesis of (5.2). Thus $x^{2} B=\mathscr{D}(B, A)=J(X)$ by (5.3) and (5.4). Set $\partial=\partial / \partial t \in \mathscr{D}(B)$ and set $x^{\prime}=\partial x / \partial t$. As $x$ has distinct roots, $x^{\prime}$ becomes a unit in $B / x B$. Choose $f \in B$ such that $f x^{\prime}-1 \in x B$. Similarly, $x x^{\prime \prime}-\left(x^{\prime}\right)^{2}$ becomes a unit in $B / x B$, and hence in $B / x^{2} B$. Choose $g \in B$ such that $g\left(x x^{\prime \prime}-\left(x^{\prime}\right)^{2}\right)-1 \in x^{2} B$. An elementary computation shows that $1, x, x f \partial, h\left(x \partial^{2}-x^{\prime} \partial\right) \in \mathscr{D}(A)$, and by a degree argument their images are linearly independent in $\mathscr{D}(A) / x^{2} \mathscr{D}(B)$. One sees that $\operatorname{dim}_{k} H(X) \geqslant 4$. 
But as $\operatorname{dim}_{k} \mathcal{O}(X) / x^{2} B=2,(5.4)$ implies that

$$
H(X) \subseteq \operatorname{End}_{k} \mathcal{O}(X) / I \cong M_{2}(k)
$$

the ring of $2 \times 2$ matrices over $k$. Thus $H(X) \cong M_{2}(k)$.

Remark. An amusing consequence of the above example is that, for the ideal $\mathfrak{m}=k x+x^{2} B$, one can distinguish elements of $\mathfrak{m} / \mathfrak{m}^{2}$ by their properties with respect to differential operators. To see this, observe that $\left\{x, x^{2} t, \ldots, x^{2} t^{n-1}\right\}$ give a basis for $\mathrm{m} / \mathrm{m}^{2}$, where $n=\operatorname{deg}(x)$. As the $\mathscr{D}(A)$-module $A$ has a unique non-zero submodule $x^{2} B$ (this follows from the fact that $x^{2} \mathscr{D}(B)$ is the unique proper ideal of $\mathscr{D}(A))$, there exists $D \in \mathscr{D}(A)$ such that $D * x=1$. In contrast, for all $E \in \mathscr{D}(A), E * x^{2} t \in x^{2} B$.

5.6. We can now construct our main example to show that $\mathscr{D}(X)$ may have infinitely many ideals.

Proposition. There exists a curve $X$ such that

$$
H(X) \cong\left(\begin{array}{cc}
k & 0 \\
k^{2} & k
\end{array}\right) .
$$
In particular, for each one-dimensional subspace $V$ of $k^{2}$, the set $\left(\begin{array}{ll}0 & 0 \\ V & 0\end{array}\right)$
is an ideal of $H(X)$.

Proof (Notation (5.2)). Take $B=k[t]$, and $x \in B$ a product of three (or more) distinct linear factors (that is, each root occurs with multiplicity one). Write $A=k+k x+k x t+x^{2} B$. Once again $J(X)=x^{2} \mathscr{D}(B)$, where $\mathscr{O}(X)=A$. Choose $f \in B$ such that $f x^{\prime}-1 \in x B$. An elementary computation shows that $1, x, x t, x f \partial \in \mathscr{D}(A)$, and their images in $\mathscr{D}(A) / x^{2} \mathscr{D}(B)$ are linearly independent. To show that these elements span $\mathscr{D}(A) / x^{2} \mathscr{D}(B)$ seems to require rather unpleasant calculations, and we will only outline the argument leaving the details to the reader.

Observe that as $\pi: \tilde{X} \rightarrow X$ is unramified at all points, $\mathscr{D}(A) \subseteq \mathscr{D}(B)$. By (5.3) we have $\mathscr{D}(B, A)=x^{2} \mathscr{D}(B)$, and hence in the notation of $(1.5), \mathscr{D}(A) \subseteq$ $\mathbf{I}\left(x^{2} \mathscr{D}(B)\right)$. One now shows that

$$
\mathbf{I}\left(x^{2} \mathscr{D}(B)\right)=B+B x \partial+B\left(x \partial^{2}-x^{\prime} \partial\right)+x^{2} \mathscr{D}(B) .
$$

To see this, check that the right-hand side really does belong to the idealiser, and then compare the dimension of both sides modulo $x^{2} \mathscr{D}(B)$; as $x$ is a product of distinct linear factors, (4.15) shows that $\operatorname{dim}_{k} \mathbf{I}\left(x^{2} \mathscr{D}(B)\right) / x^{2} \mathscr{D}(B)=4 \operatorname{deg} x$, and it is straightforward to check that the same dimension occurs on the right-hand side.

It is obvious that $\operatorname{Der}_{k} A \subseteq B x \partial$, and to compute $\operatorname{Der}_{k} A$ precisely note that if $b x \partial \in \operatorname{Der}_{k} A$, then evaluation at $x, x t \in A$ gives $b x x^{\prime}, b x x^{\prime} t \in A$. This can only occur if $b \in k f+B x$ (where $f$ is as above). Hence $\mathscr{D}^{1}(A)=A+k x f \partial+B x^{2} \partial$. Finally, we need to show that

$$
\mathscr{D}^{2}(A) \subseteq A+k x f \partial+B x^{2} \partial+B x^{2} \partial^{2} .
$$

To see this, note that if $D=b\left(x \partial^{2}-x^{\prime} \partial\right)+c x \partial \in \mathscr{D}(A)$ for some $b, c \in B$, then certainly $D * A \subseteq k+x B$, whence $b \in x B$ and so $b x \partial^{2} \in x^{2} \mathscr{D}(B) \subseteq \mathscr{D}(A)$. Thus $D \in B x^{2} \partial^{2}+\mathscr{D}^{1}(A)$. Putting all these observations together shows that $H(X)$ is spanned by the images of $1, x, x t, x f \partial$. 
To give a ring-theoretic description of $H(X)$, consider the action of $H(X)$ on the faithful $H(X)$-module $A / x^{2} B$. The images of $1, x, x t$ give a basis for $A / x^{2} B$. Describing $H(X)$ in terms of the usual matrix units with respect to this basis gives $x \rightarrow e_{21}, x t \rightarrow e_{31}, x f \partial \rightarrow e_{22}+e_{33}$. Thus

$$
H(X)=\left\{\left(\begin{array}{ccc}
\alpha & 0 & 0 \\
\beta & \delta & 0 \\
\gamma & 0 & \delta
\end{array}\right) \mid \alpha, \beta, \gamma, \delta \in k\right\} \subseteq M_{3}(k),
$$

and this ring is isomorphic to $\left(\begin{array}{cc}k & 0 \\ k^{2} & k\end{array}\right)$.

REMARK. Note that gl.dim $H(X)=1$. It can be shown that $2 \leqslant g l \cdot d i m \mathscr{D}(X) \leqslant 3$.

5.7. In all the examples considered so far it should be noticed that if $\mathscr{D}(\tilde{X}, X) * \mathscr{O}(\tilde{X}) \neq \mathscr{O}(X)$ then $\mathscr{D}(\tilde{X}, X) * \mathscr{O}(\tilde{X})$ is actually equal to the conductor of $\mathcal{O}(X)$ in $\mathcal{O}(\tilde{X})$. This need not be the case, as the following illustrates.

Example. Set $B=k[t]=\mathcal{O}\left(\mathbf{A}^{1}\right)$. Define $X$ to be the curve given by

$$
\mathcal{O}(X)=k+k t^{2}(t-1)+t^{4}(t-1) B .
$$

Write $A=\mathcal{O}(X)$. The conductor of $\mathcal{O}(X)$ in $\mathcal{O}(\tilde{X})=\mathcal{O}\left(\mathbf{A}^{1}\right)$ is equal to $t^{4}(t-1) B$. Let $\mathfrak{m}=k t^{2}(t-1)+t^{4}(t-1) B$. Then $\mathscr{D}(\tilde{X}, X) * \mathscr{O}(\bar{X})=\mathfrak{m}$.

Proof. Since $\mathfrak{m}$ is contained in two distinct maximal ideals of $B$, namely $t B$ and $(t-1) B$, the normalisation map $\pi: \bar{X} \rightarrow X$ is not injective. By (3.7) and (3.3) it follows that $\mathscr{D}(\bar{X}, X) * \mathscr{O}(\bar{X}) \neq \mathscr{O}(X)$.

Consider $D=t^{2}(t-1)(t \partial-1) \in \mathscr{D}(\tilde{X})$. An easy computation shows that $D \in$ $\mathscr{D}(\tilde{X}, X)$ and $D *(-1)=t^{2}(t-1) \in \mathscr{D}(\tilde{X}, X) * \mathscr{O}(\tilde{X})$. But also $t^{4}(t-1) \epsilon$ $\mathscr{D}(\tilde{X}, X)$. Thus $\mathfrak{m} \subseteq \mathscr{D}(\tilde{X}, X) * \mathscr{O}(\tilde{X})$. Hence one has equality.

Remarks. (1) It is easy to show that $\mathscr{D}(\tilde{X}, X)=D \mathscr{D}(\tilde{X})+t^{4}(t-1) \mathscr{D}(\tilde{X})$ for this example.

(2) Since $\mathcal{O}(X) / \mathscr{D}(\tilde{X}, X) * \mathscr{O}(\bar{X})$ is a faithful simple $H(X)$-module, one obtains $H(X)=k$ for this example.

(3) As the normalisation $\pi: \tilde{X} \rightarrow X$ is ramified at the origin, $\mathscr{D}(X) \nsubseteq \mathscr{D}(\tilde{X})$ by (3.6). Combined with (2) this also implies that $J(X) \neq \mathscr{D}(\bar{X}, X)$. An operator in $\mathscr{D}(X)$ but not in $\mathscr{D}(\bar{X})$ is $t^{-1}(t-1)(t \partial-1)(t \partial-2)(t \partial-4) \partial$.

5.8. Consider the situation of Theorem 4.11 and the remark following it. Factorise the normalisation map $\tilde{X} \stackrel{\psi}{\longrightarrow} Y \stackrel{\varphi}{\longrightarrow} X$ with $\psi$ unramified at all points, and $\varphi$ injective. The following example illustrates that $\mathscr{D}(X)$ and $\mathscr{D}(Y)$ need not be Morita equivalent.

Set $B=k[t], \mathcal{O}(Y)=k+x B, \mathcal{O}(X)=k+k x+k x t+x^{2} B$ where $x \in B$ has distinct roots and $\operatorname{deg} x \geqslant 3$. By (4.11), $H(Y) \cong k$, and by (5.6),

$$
H(X) \cong\left(\begin{array}{cc}
k & 0 \\
k^{2} & k
\end{array}\right)
$$


Since the lattices of two-sided ideals in $\mathscr{D}(X)$ and $\mathscr{D}(Y)$ are different, $\mathscr{D}(X)$ and $\mathscr{D}(Y)$ cannot be Morita equivalent.

\section{Projective curves}

6.1. Let $C$ be a projective curve with normalisation $\tilde{C}$, and denote by $\pi: \bar{C} \rightarrow C$ the natural projection. Let $\mathscr{D}_{C}-$ Mod denote the category of sheaves of quasi-coherent $\mathscr{D}_{C}$-modules, similarly for $\mathscr{D}_{\tilde{\mathrm{C}}^{-}} M_{0}$.

Theorem. Suppose that $\pi: \widetilde{C} \rightarrow C$ is injective. Then the categories $\mathscr{D}_{\mathrm{C}}-$ Mod and $\mathscr{D}_{\tilde{\mathrm{C}}}$-Mod are equivalent.

Proof. Note that $\pi$ is an affine morphism. Let $\pi_{*}$ denote the direct image functor, and consider $\pi_{*} \mathscr{D}_{\bar{C}}$. As $\mathscr{D}_{\bar{C}}$ is a quasi-coherent $\mathscr{O}_{\bar{C}}$-module, and $\pi_{*} \mathscr{O}_{\bar{C}}$ is a quasi-coherent $\mathscr{O}_{\mathrm{C}}$-module, it follows that $\pi_{*} \mathscr{D}_{\bar{C}}$ is a quasi-coherent $\mathscr{O}_{C}$-module. Write $\pi_{*} \mathscr{D}_{\bar{C}}-M_{0} d$ for the category of $\pi_{*} \mathscr{D}_{\tilde{C}}$-modules which are quasi-coherent as $\hat{O}_{C}$-modules (we may view $\mathscr{O}_{C}$ as a subsheaf of $\pi_{*} \mathscr{O}_{\bar{C}}$ ). Then, by an argument along the same lines as [10, Chapter II, Ex. 5.17(e)], $\pi_{*} \mathscr{D}_{\bar{C}}-M_{o d}$ and $\mathscr{D}_{\bar{C}}-M_{\text {ad }}$ are equivalent categories.

Write $\mathscr{P}=\mathscr{D}_{C}\left(\pi_{*} \mathscr{O}_{\bar{C}}, \mathscr{O}_{C}\right)$; that is, $\mathscr{P}$ is the sheaf on $C$ with sections over an open affine $U \subseteq C$ consisting of those differential operators on $\left(\pi_{*} \mathscr{O}_{\bar{C}}\right)(U)=$ $\mathscr{O}_{\bar{c}}\left(\pi^{-1}(U)\right.$ ) which send $\mathscr{O}_{\bar{C}}\left(\pi^{-1}(U)\right)$ into $\mathscr{O}_{C}(U)$ (which we view as a subalgebra of $\left.\tilde{O}_{\tilde{C}}\left(\pi^{-1}(U)\right)\right)$. Then $\mathscr{P}$ is a left $\mathscr{D}_{C}$-module, and a right $\pi_{*} \mathscr{D}_{\bar{C}}$-module. After Theorem 3.4, there is an open affine cover $U_{\lambda}$ for $C$ such that each $\mathscr{P}\left(U_{\lambda}\right)$ is a progenerator in $\mathscr{D}_{C}\left(U_{\lambda}\right)-M_{o d}$. Put $\mathscr{P}^{-1}=\mathscr{H}_{a m_{D_{C}}}\left(\mathscr{P}, \mathscr{D}_{C}\right)$ and $\mathscr{S}=\mathscr{L}_{a m_{D_{c}}}(\mathscr{P}, \mathscr{P})$.

There is a natural morphism $\mathscr{P}^{-1} \otimes_{\mathscr{D}_{C}} \mathscr{P} \rightarrow \mathscr{S}$ given by $(q \otimes p)(w)=q(p) w$ for $q \in \mathscr{P}^{-1}, p \in \mathscr{P}, w \in \mathscr{P}$. This is locally an isomorphism and hence globally (remember that $\mathscr{P}$ is locally free as a $\mathscr{D}_{C}$-module, with inverse $\mathscr{P}^{-1}$ ). Also the natural morphism $\mathscr{P} \otimes_{\mathscr{S}} \mathscr{P}^{-1} \rightarrow \mathscr{D}_{C}$ is locally an isomorphism by Theorem 3.4, and hence globally. It is now clear that the functors

$$
\mathscr{P} \otimes_{\mathscr{S}}-: \mathscr{S}_{-} \operatorname{Mod} \rightarrow \mathscr{D}_{C}-M_{o d}
$$

and

$$
\mathscr{P}^{-1} \otimes_{\mathscr{D}_{C}}-: \mathscr{D}_{C}-M_{o d} \rightarrow \mathscr{S}-M_{o d}
$$

are mutually inverse, and give an equivalence between these two categories.

To complete the proof observe that $\pi_{*} \mathscr{D}_{\bar{C}} \cong \mathscr{S}$. To see this, first note that there is a morphism $\pi_{*} \mathscr{D}_{\bar{C}} \rightarrow \mathscr{S}$ because $\mathscr{P}$ is a $\mathscr{D}_{C}-\pi_{*} \mathscr{D}_{\bar{C}}$ bimodule. This is locally an isomorphism since for all open affine $U \subseteq \tilde{C}, \pi_{*} \mathscr{D}_{\tilde{C}}(U)=\mathscr{D}(U)$ is a simple ring. Hence there is a global isomorphism $\pi_{*} \mathscr{D}_{\bar{C}} \rightarrow \mathscr{S}$.

\section{Higher-dimensional varieties}

A famous example due to Bernstein, Gelfand, and Gelfand [3] shows that the ring of differential operators on an affine variety of dimension at least 2 need not be finitely generated or noetherian. Their example rests ultimately on properties of the cohomology of sheaves on an elliptic curve. In this section we show that our techniques for curves can be applied to certain varieties of dimension at least 2 to obtain other examples of non-noetherian rings of differential operators. 
Throughout this section $X$ will denote a singular variety over $k$ of dimension at least 2, with the property that $\tilde{X}$, the normalisation of $X$, is non-singular. If $X$ is regular in codimension 1 , we show that $\mathscr{D}(X)$ is not left noetherian. In contrast, however, if Sing $X$ is finite then $\mathscr{D}(X)$ is right noetherian, and a finitely generated $k$-algebra. Thus $\mathscr{D}(X)$ can display a curious lack of symmetry.

7.2. Part (a) of the following appears in [2, Proposition 1], but we include a (different) proof since it is both easy and amusing.

Proposition. Let $X$ be a singular variety of dimension $n \geqslant 2$, and suppose that $\tilde{X}$ is non-singular. Suppose that $X$ is regular in codimension 1 . Then

(a) $\mathscr{D}(X) \subseteq \mathscr{D}(\tilde{X})$,

(b) $\mathscr{D}(X)$ is not a simple ring.

Proof. Write $A=\mathscr{O}(X), B=\mathscr{O}(\tilde{X})$, and $I=\operatorname{Ann}_{A}(B / A)$. As $X$ is non-singular in codimension $1, \operatorname{dim}(A / I) \leqslant n-2$ (where dim denotes Krull dimension). As $P=\mathscr{D}(\tilde{X}, X) \supseteq I$, it follows that $d(\mathscr{D}(\tilde{X}) / P) \leqslant 2 n-2$ (notation (1.4(f))). Hence by $\left[4\right.$, Chapter 2, Theorem 7.1], $\operatorname{Ext}^{1}(\mathscr{D}(\tilde{X}) / P, \mathscr{D}(\tilde{X}))=0$. Write $P^{*}=$ $\{q \in$ Fract $\mathscr{D}(\tilde{X}) \mid q P \subseteq \mathscr{D}(\tilde{X})\}$. Apply the functor $\operatorname{Hom}_{\mathscr{D}(\tilde{X})}(-, \mathscr{D}(\tilde{X}))$ to the sequence

$$
0 \rightarrow P \rightarrow \mathscr{D}(\tilde{X}) \rightarrow \mathscr{D}(\tilde{X}) / P \rightarrow 0
$$

to obtain $\mathscr{D}(\tilde{X})=P^{*}$, since the $\operatorname{Ext}^{1}$ group vanishes. However, $\mathscr{D}(X) \subseteq P^{*}$, which gives (a). Part (b) follows from the fact that $\mathscr{D}(\bar{X}, X)$ is now a proper ideal of $\mathscr{D}(X)$.

7.3. Proposition. Let $X$ be a singular variety with non-singular normalisation $\tilde{X}$. Suppose that $\operatorname{Sing} X$ is finite. Then $\mathscr{D}(X)$ is a finitely generated right noetherian $k$-algebra.

Proof. If $X$ is a curve this is just Theorem 2.5. Suppose that $\operatorname{dim} X=n \geqslant 2$. By (7.2), $\mathscr{D}(X) \subseteq \mathscr{D}(\tilde{X})$. Since Sing $X$ is finite, $\operatorname{dim}_{k} \mathscr{O}(\tilde{X}) / \mathscr{O}(X)<\infty$, and $\operatorname{dim}_{k} O(X) / I<\infty$ where $I$ is the conductor of $\mathscr{O}(X)$ in $O(\bar{X})$. Thus $d(\mathscr{D}(\tilde{X}) / I \mathscr{D}(\tilde{X}))=n$, and by $(1.4(\mathrm{f})), \mathscr{D}(\tilde{X}) / I \mathscr{D}(\tilde{X})$ is of finite length. However, $I \mathscr{D}(\bar{X}) \subseteq \mathscr{D}(\tilde{X}, X)$ and $\mathscr{D}(\bar{X}, X)$ is a two-sided ideal of $\mathscr{D}(X)$. Thus $\mathscr{D}(\bar{X}) / \mathscr{D}(\tilde{X}, X)$ is of finite length, and $\mathscr{D}(X) \subseteq \mathbf{I}(\mathscr{D}(\tilde{X}, X))$. But now by (1.5), $\operatorname{dim}_{k} \mathscr{D}(X) / \mathscr{D}(\bar{X}, X)<\infty$. By [20, Proposition 1], it follows that $\mathscr{D}(X)$ is right noetherian. Further, since $\mathscr{D}(\tilde{X})$ is finitely generated, it follows from [20, Proposition 2(b)] that $\mathscr{D}(X)$ is finitely generated.

Remark. For some special cases when $\mathscr{D}_{X, x}$ is generated by $\mathscr{O}_{X, x}$ and a finite number of differential operators (in the analytic case) see [26, Theorem 6].

7.4. Before constructing our non-noetherian rings of differential operators, we need the following routine extension of [10, Chapter II, Theorem 8.8] for which we have been unable to find a reference in the literature.

Proposition. Let $A$ be a regular, semi-local ring of finite type over $k$. Suppose, 
for each of the maximal ideals $\mathfrak{m}_{1}, \ldots, \mathfrak{m}_{r}$ of $A$, that $A / \mathfrak{m}_{i} \cong k$ and $\operatorname{dim} A_{\mathfrak{m}}=n=$ $\operatorname{dim} A$. Then there exist $t_{1}, \ldots, t_{n} \in J(A)$, the Jacobson radical of $A$, and $\partial_{1}, \ldots, \partial_{n} \in \operatorname{Der}_{k} A$ such that $\partial_{i}\left(t_{j}\right)=\delta_{i j}$ for all $1 \leqslant i, j \leqslant n$.

Proof. By [10, Chapter II, Theorem 8.15], $\operatorname{Der}_{k} A$ is a projective $A$-module of rank $n$. Since $A$ is semi-local, it follows that $\operatorname{Der}_{k} A$ is actually free, say with basis $\partial_{1}, \ldots, \partial_{n}$. For any $i \leqslant n$ and $j \leqslant r$, let $\bar{\partial}_{i}: \mathfrak{m}_{j} / \mathfrak{m}_{j}^{2} \rightarrow A / \mathfrak{m}_{j}$ be the map induced by $\partial_{i}$. Since $\operatorname{Der}_{k}\left(A_{\mathrm{m}}\right) \cong A_{\mathrm{m}} \otimes_{A} \operatorname{Der}_{k} A$, the $\partial_{i}$ are also a basis for $\operatorname{Der}_{k}\left(A_{\mathrm{m}}\right)$. Thus the $\bar{\partial}_{i}$ form a $k$-basis for $\operatorname{Hom}_{k}\left(\mathfrak{m}_{j} / \mathfrak{m}_{j}^{2}, A / \mathfrak{m}_{j}\right)$. Choose $\bar{t}_{i j} \in \mathfrak{m}_{j} / \mathfrak{m}_{j}^{2}$ such that $\bar{\partial}_{k}\left(\bar{t}_{i j}\right)=\delta_{k i}$. For $1 \leqslant i \leqslant n$, set

$$
\bar{t}_{i}=\left(\bar{t}_{i 1}, \ldots, \bar{t}_{i r}\right) \in\left(\mathfrak{m}_{1} / \mathfrak{m}_{1}^{2}\right) \bigoplus \ldots \oplus\left(\mathfrak{m}_{r} / \mathfrak{m}_{r}^{2}\right) \cong J(A) / J(A)^{2} .
$$

Then $\bar{\partial}_{k}\left(\bar{t}_{i}\right)=\delta_{k i} \in A / J(A)$. Let $t_{i}$ be any inverse image of $\bar{t}_{i}$ in $A$. Then we have shown that $\partial_{k}\left(t_{i}\right) \equiv \delta_{i k}(\bmod J(A))$. Thus the matrix $B=\left(\partial_{k}\left(t_{i}\right)\right)$ has determinant congruent to $1(\bmod J(A))$, and so is invertible. Thus setting $\left(\gamma_{1}, \ldots, \gamma_{n}\right)^{T}=$ $B^{-1}\left(\partial_{1}, \ldots, \partial_{n}\right)^{T}$ we have $\gamma_{i} \in \operatorname{Der}_{k} A$ for $1 \leqslant i \leqslant n$ and $\gamma_{i}\left(t_{j}\right)=\delta_{i j}$, as required.

7.5. We are now ready to give the main result of this section, by providing a large number of varieties $X$, such that $\mathscr{D}(X)$ is not left noetherian. The idea behind this proof was motivated by an observation of R. Resco [19] showing that idealisers in the second Weyl algebra are often non-noetherian.

THEOREM. Let $X$ be a singular variety with non-singular normalisation $\bar{X}$. Suppose that $X$ is non-singular in codimension 1 . Then $\mathscr{D}(X)$ is not left noetherian.

Remark. This also implies that gr $\mathscr{D}(X)$ is not noetherian, and hence not finitely generated. For some special cases of this see [26].

Proof. Set $n=\operatorname{dim} X$. Pick $x \in X$ a singular point with corresponding maximal ideal $\mathrm{m} \subseteq \mathscr{O}(X)$. Set $A=\mathscr{O}_{X, x} \subseteq B=\mathscr{O}_{\tilde{X}, \pi^{-1}(x)}$. Since $\mathscr{D}(A)=\mathscr{D}(X) \otimes_{\mathcal{O}(X)} \mathscr{O}_{X, x}$, it clearly suffices to prove that $\mathscr{D}(A)$ is not left noetherian. As $B$ is a regular, semi-local ring, it follows from (7.4) that there exist $t_{1}, \ldots, t_{n} \in B$ and $\partial_{1}, \ldots, \partial_{n} \in \operatorname{Der}_{k} B$ such that $\partial_{i} * t_{j}=\delta_{i j}$ for all $i, j$.

Set $I=\operatorname{Ann}_{A}(B / A)$. Since $X$ is regular in codimension 1, the height of $I$ is at least 2. Hence there exists $0 \neq f \in I \cap k\left[t_{1}, \ldots, t_{n}\right]$ (if not then $k\left[t_{1}, \ldots, t_{n}\right]$ embeds in $B / I$, contradicting the fact that $\left.\operatorname{tr} \cdot \operatorname{deg}_{k}(B / I) \leqslant n-2\right)$. By an elementary change of variables, we may assume that $f$ is monic as a polynomial in $t_{n}$ with coefficients in $k\left[t_{1}, \ldots, t_{n-1}\right]$. Again, as height $(I) \geqslant 2$, there exists $0 \neq g \in I \cap k\left[t_{1}, \ldots, t_{n-1}\right]$. Write $P=\mathscr{D}(B, A)$ and $\partial=\partial_{n}$.

Suppose that $\mathscr{D}(A)$ is left noetherian. Then, as $g \partial^{i} \in P$, the module $M=$ $\sum_{i=0}^{\infty} \mathscr{D}(A) g \partial^{i}$ is a finitely generated left ideal of $\mathscr{D}(A)$. Hence, as $g \partial=\partial g$, $N=\sum_{i=0}^{\infty} \mathscr{D}(A) \partial^{i}$ is a finitely generated left $\mathscr{D}(A)$-module. Thus for some $m \geqslant 1$, and some $r_{i} \in \mathscr{D}(A), \partial^{m}+\sum_{i=0}^{m-1} r_{i} \partial^{i}=0$. The idea now is to mimic the obvious (computational) proof that, for the Weyl algebra $\mathscr{D}\left(\mathbf{A}^{n}\right)$,

$$
t_{n} \mathscr{D}\left(\mathbf{A}^{n}\right)+\left(\partial / \partial t_{n}\right)^{m} \mathscr{D}\left(\mathbf{A}^{n}\right)=\mathscr{D}\left(\mathbf{A}^{n}\right)
$$

in order to prove that $P=\mathscr{D}(B)$ (so obtaining a contradiction). The relevant computations are contained in the following sublemma. 
Sublemma. Let $z, w \in k\left[t_{1}, \ldots, t_{n}\right] \subseteq \mathscr{D}(B)$, and $u, s \in \mathbf{N}$. If $m$ is as in the proof above, then

(i) for $0 \leqslant i \leqslant m-1, \partial^{i} z^{u+m} w^{s+m} \in z^{u+1} w^{s} \mathscr{D}(B)$;

(ii) there exists $0 \neq \lambda \in k$ such that

$$
\partial^{m} z^{u+m} w^{s+m} \in \lambda z^{u} w^{s+m}(\partial * z)^{m}+z^{u+1} w^{s} \mathscr{D}(B) ;
$$

(iii) if $z^{q} \in P$ for some $q \geqslant 1$, then $(\partial * z)^{2 m q} \in P$.

Proof of the Theorem from the Sublemma. Recall that $f \in P \cap k\left[t_{1}, \ldots, t_{n}\right]$ is monic as a polynomial in $t_{n}$, of degree $r$, say. By (iii) and an induction argument, $\left(\partial^{i} * f\right)^{(2 m)^{i}} \in P$ for $1 \leqslant i \leqslant r$. But $\partial^{r} * f=r !$; thus $1 \in P \subseteq \mathscr{D}(A)$. As $P$ is a right ideal of $\mathscr{D}(B)$, this implies that $\mathscr{D}(A)=\mathscr{D}(B)$, contradicting the fact that $A \neq B$. Hence $\mathscr{D}(A)$ (and consequently $\mathscr{D}(X)$ ) is not left noetherian.

It therefore remains to provide:

Proof of the Sublemma. Parts (i) and (ii) are routine consequences of the fact that $\partial z^{j}=z^{j} \partial+j(\partial * z) z^{j-1}$. Part (iii) is proved by induction. Write $w=\partial * z$. Suppose that $z^{q-v} w^{2 m v} \in P$ for some $0 \leqslant v<q$ (by hypothesis this does hold for $v=0)$. Since $P \mathscr{D}(B) \subseteq P, \quad z^{q-v+(m-1)} w^{2 m v+m} \in P$. Remember that $\partial^{m}+\sum_{i=0}^{m-1} r_{i} \partial^{i}=0$. Thus by (i) and (ii),

$$
\begin{aligned}
0 & =\left[\partial^{m}+\sum_{i=0}^{m-1} r_{i} \partial^{i}, z^{(q-v-1)+m} w^{2 m v+m}\right] \\
& \in \lambda z^{q-v-1} w^{2 m v+m} w^{m}+\sum_{i=0}^{m-1} r_{i} z^{q-v} w^{2 m v} \mathscr{D}(B),
\end{aligned}
$$

where $0 \neq \lambda \in k$. Since for each $i, r_{i} z^{q-v} w^{2 m v} \mathscr{D}(B) \subseteq \mathscr{D}(A) P \mathscr{D}(B) \subseteq P$, this implies that $\lambda z^{q-v-1} w^{2 m v+m} \in P$. This completes the inductive step, and so by induction, $w^{2 m q} \in P$.

7.6. The following summarises the local versions of the results in this section-the proofs are essentially the same as those already given.

Theorem. Let $X$ be an irreducible variety, and $x \in X$ a singular point. Suppose that the normalisation at $x$ is regular. Then

(a) if all height-1 primes of $\mathcal{O}_{X, x}$ are regular (and hence $\operatorname{dim} X \geqslant 2$ ) then

(i) $\mathscr{D}_{X, x} \subseteq \mathscr{D}_{\bar{X}_{, \pi^{-1}}(x)}$,

(ii) $\mathscr{D}_{X, x}$ is not a simple ring,

(iii) $\mathscr{D}_{X, x}$ is not left noetherian;

(b) if $x$ is an isolated singularity then $\mathscr{D}_{X, x}$ is right noetherian and is generated by $\mathcal{O}_{X, x}$ and a finite number of differential operators.

\section{References}

1. F. W. ANDERson and K. R. Fuller, Rings and categories of modules, Graduate Texts in Mathematics 13 (Springer, Berlin, 1974).

2. J. BECKER, 'Higher derivations and integral closure', Amer. J. Math. 100 (1978) 495-521.

3. J. N. Bernstein, I. M. Gelfand, and S. I. Gelfand, 'Differential operators on the cubic cone', Russian Math. Surveys 27 (1972) 169-174. 
4. J. E. BJORK, Rings of differential operators, North-Holland Mathematical Library (NorthHolland, Amsterdam, 1979).

5. T. Bloом, 'Differential operators on curves', Rice Univ. Stud. 59 (1973) 13-19.

6. W. C. BROWN, 'A note on higher derivations and ordinary points of curves', Rocky Mountain J. Math. 14 (1984) 397-402.

7. S. U. CHASE, 'On the homological dimension of algebras of differential operators', Comm. Algebra 5 (1974) 351-363.

8. K. GOODEARL, 'Subrings of idealiser rings', J. Algebra 33 (1975) 405-429.

9. A. GRothendieCK, Eléments de géométrie algébrique IV, Publications Mathématiques 32 (Institut des Hautes Études Scientifiques, Paris, 1967).

10. R. Hartshorne, Algebraic geometry, Graduate Texts in Mathematics 52 (Springer, Berlin, 1977).

11. R. G. Heynemann and M. Sweedler, 'Affine Hopf algebras', J. Algebra 13 (1969) 192-241.

12. T. H. Lenagan, 'Gelfand-Kirillov dimension and affine PI rings', Comm. Algebra 10 (1982) 87-92.

13. B. Malgrange, 'Les éspaces analytiques', Enseign. Math. 14 (1968) 1-28.

14. H. Matsumura, Commutative algebra (W. A. Benjamin, New York, 1970).

15. S. M. MONTGOMERY and L. W. SMall, 'Fixed rings of Noetherian rings', Bull. London Math. Soc. 13 (1981) 33-38.

16. J. MUHASKY, 'The differential operator ring of an affine curve', Ph.D. Thesis, University of Utah, 1986.

17. I. Musson, 'Some rings of differential operators which are Morita equivalent to the Weyl algebra $A_{1}$ ', Proc. Amer. Math. Soc. 98 (1986) 29-30.

18. D. QUILLEN, 'On the endomorphism ring of a simple module over an enveloping algebra', Proc. Amer. Math. Soc. 21 (1969) 171-172.

19. R. RESCO, 'Affine domains of finite Gelfand-Kirillov dimension which are right, but not left, noetherian', Bull. London Math. Soc. 16 (1984) 590-594.

20. J. C. RobSON and L. W. Small, 'Orders equivalent to the first Weyl algebra', Quart. J. Math. Oxford 37 (1986) 475-482.

21. J. P. SERRE, Groupes algébriques et corps de classes (Hermann, Paris, 1959).

22. S. P. SMITH, 'An example of a ring Morita equivalent to the Weyl algebra', J. Algebra 73 (1981) 552-555.

23. S. P. SMITH, 'Gelfand-Kirillov dimension of rings of formal differential operators on affine varieties', Proc. Amer. Math. Soc. 90 (1984) 1-8.

24. J. T. STAFFORD, 'Endomorphism rings of right ideals of the Weyl algebra', Trans. Amer. Math. Soc. 299 (1987) 623-639.

25. M. E. SwEEDLER, Groups of simple algebras, Publications Mathématiques 44 (Institut des Hautes Études Scientifiques, Paris, 1975).

26. J. P. VIGUÉ, 'Opérateurs différentiels sur les espaces analytiques', Invent. Math. 20 (1973) 313-336.

\section{Mathematics Institute University of Warwick Coventry CV4 7AL}

\author{
Department of Pure Mathematics \\ University of Leeds \\ Leeds LS2 9JT
}

